\title{
Schur-Weyl duality for orthogonal groups
}

\author{
Stephen Doty and Jun Hu
}

\begin{abstract}
We prove Schur-Weyl duality between the Brauer algebra $\mathfrak{B}_{n}(m)$ and the orthogonal group $O_{m}(K)$ over an arbitrary infinite field $K$ of odd characteristic. If $m$ is even, we show that each connected component of the orthogonal monoid is a normal variety; this implies that the orthogonal Schur algebra associated to the identity component is a generalized Schur algebra. As an application of the main result, an explicit and characteristic-free description of the annihilator of $n$-tensor space $V^{\otimes n}$ in the Brauer algebra $\mathfrak{B}_{n}(m)$ is also given.
\end{abstract}

\section{Introduction}

Let $m, n \in \mathbb{N}$. Write $\lambda \vdash n$ to mean that $\lambda=\left(\lambda_{1}, \lambda_{2}, \ldots\right)$ is a partition of $n$, and denote by $\ell(\lambda)$ the largest integer $i$ such that $\lambda_{i} \neq 0$.

Let $K$ be an infinite field and $V$ an $m$-dimensional $K$-vector space. The natural left action of the general linear group $G L(V)$ on $V^{\otimes n}$ commutes with the right permutation action of the symmetric group $\mathfrak{S}_{n}$. Let $\varphi, \psi$ be the corresponding natural representations

$$
\varphi:\left(K \mathfrak{S}_{n}\right)^{\mathrm{op}} \rightarrow \operatorname{End}_{K}\left(V^{\otimes n}\right), \quad \psi: K G L(V) \rightarrow \operatorname{End}_{K}\left(V^{\otimes n}\right),
$$

respectively. The well-known Schur-Weyl duality (see [8], [17, [37, [46, [47]) says that

(a) $\varphi\left(\left(K \mathfrak{S}_{n}\right)^{\mathrm{op}}\right)=\operatorname{End}_{K G L(V)}\left(V^{\otimes n}\right)$, and if $m \geq n$ then $\varphi$ is injective, and hence an isomorphism onto $\operatorname{End}_{K G L(V)}\left(V^{\otimes n}\right)$,

(b) $\psi(K G L(V))=\operatorname{End}_{K \mathfrak{S}_{n}}\left(V^{\otimes n}\right)$,

(c) if char $K=0$, then there is an irreducible $K G L(V)-K \mathfrak{S}_{n}$-bimodule decomposition

$$
V^{\otimes n}=\bigoplus_{\substack{\lambda=\left(\lambda_{1}, \lambda_{2}, \cdots\right) \vdash n \\ \ell(\lambda) \leq m}} \Delta_{\lambda} \otimes S^{\lambda}
$$

where $\Delta_{\lambda}$ (resp., $S^{\lambda}$ ) denotes the irreducible $K G L(V)$-module (resp., irreducible $K \mathfrak{S}_{n^{-}}$ module) associated to $\lambda$.

There are also Schur-Weyl dualities for symplectic groups and orthogonal groups in the semisimple case, i.e., when $K$ has characteristic zero; see [3], 4] and [5]. In these cases, the symmetric group will be replaced by certain specialized Brauer algebras. We are mostly interested in the non-semisimple case. In [10, Schur-Weyl duality between the Brauer algebra $\mathfrak{B}_{n}(-2 m)$ and the symplectic group $\operatorname{Sp}_{2 m}(K)$ over an arbitrary infinite field $K$ was proved. In [30], the second author gave an explicit and characteristic-free description of the annihilator of $n$-tensor space $V^{\otimes n}$ in the Brauer algebra $\mathfrak{B}_{n}(-2 m)$.

The aim of this work is to generalize these results to the orthogonal case. We first recall the definition of orthogonal group over an arbitrary infinite field $K$ with char $K \neq 2$. Let $V$ be an

2000 Mathematics Subject Classification 20G05 (primary), 20C20 (secondary)..

The second author was supported by the National Natural Science Foundation of China (Project 10771014), the Program NCET and the Scientific Research Foundation for the Returned Overseas Chinese Scholars, State Education Ministry. 
$m$-dimensional $K$-vector space with a non-degenerate symmetric bilinear form (,). Then the orthogonal similitude group (resp., orthogonal group) relative to $($,$) is$

$$
\begin{gathered}
G O(V):=\left\{g \in G L(V) \mid \begin{array}{r}
\exists 0 \neq d \in K, \text { such that }(g v, g w)=d(v, w) \\
\forall v, w \in V
\end{array}\right\} \\
(\text { resp., } O(V):=\{g \in G L(V) \mid(g v, g w)=(v, w), \forall v, w \in V\} .)
\end{gathered}
$$

By restriction from $G L(V)$, we get natural left actions of $G O(V)$ and $O(V)$ on $V^{\otimes n}$. Note that if $0 \neq d \in K$ is such that $(g v, g w)=d(v, w)$ for any $v, w \in V$, then $\left(\left(\sqrt{d^{-1}} g\right) v,\left(\sqrt{d^{-1}} g\right) w\right)=$ $(v, w)$ for any $v, w \in V$. Therefore, if $K$ is large enough such that $\sqrt{d} \in K$ for any $d \in K$, then $g \in G O(V)$ implies that $\left(a \operatorname{id}_{V}\right) g \in O(V)$ for some $0 \neq a \in K$. In that case,

$$
\begin{aligned}
\psi(g) & =\psi\left(\left(a^{-1} \operatorname{id}_{V}\right)\left(a \operatorname{id}_{V}\right) g\right)=\psi\left(a^{-1} \operatorname{id}_{V}\right) \psi\left(\left(a \operatorname{id}_{V}\right) g\right) \\
& =\left(a^{-n} \operatorname{id}_{V \otimes n}\right) \psi\left(\left(a \operatorname{id}_{V}\right) g\right)=a^{-n} \psi\left(\left(a \operatorname{id}_{V}\right) g\right) .
\end{aligned}
$$

It follows that

$$
\psi(K O(V))=\psi(K G O(V))
$$

provided $K$ is closed under square roots.

We now recall the definition of Brauer algebra. Let $x$ be an indeterminate over $\mathbb{Z}$. The Brauer algebra $\mathfrak{B}_{n}(x)$ over $\mathbb{Z}[x]$ is a unital $\mathbb{Z}[x]$-algebra with generators $s_{1}, \cdots, s_{n-1}, e_{1}, \cdots, e_{n-1}$ and relations (see [21]):

$$
\begin{gathered}
s_{i}^{2}=1, e_{i}^{2}=x e_{i}, e_{i} s_{i}=e_{i}=s_{i} e_{i}, \quad \forall 1 \leq i \leq n-1, \\
s_{i} s_{j}=s_{j} s_{i}, s_{i} e_{j}=e_{j} s_{i}, e_{i} e_{j}=e_{j} e_{i}, \quad \forall 1 \leq i<j-1 \leq n-2, \\
s_{i} s_{i+1} s_{i}=s_{i+1} s_{i} s_{i+1}, e_{i} e_{i+1} e_{i}=e_{i}, e_{i+1} e_{i} e_{i+1}=e_{i+1}, \quad \forall 1 \leq i \leq n-2, \\
s_{i} e_{i+1} e_{i}=s_{i+1} e_{i}, e_{i+1} e_{i} s_{i+1}=e_{i+1} s_{i}, \quad \forall 1 \leq i \leq n-2 .
\end{gathered}
$$

$\mathfrak{B}_{n}(x)$ is a free $\mathbb{Z}[x]$-module with $\operatorname{rank}(2 n-1) \cdot(2 n-3) \cdots 3 \cdot 1$. For any commutative $\mathbb{Z}[x]$ algebra $R$ with $x$ specialized to $\delta \in R$, we define $\mathfrak{B}_{n}(\delta)_{R}:=R \otimes_{\mathbb{Z}[x]} \mathfrak{B}_{n}(x)$. This algebra was first introduced by Richard Brauer (see [3]) in order to describe how the $n$-tensor space $V^{\otimes n}$ decomposes into irreducible modules over the orthogonal group $O(V)$ or the symplectic group $S p(V)$, where $V$ is an orthogonal or symplectic vector space. In Brauer's original formulation, the algebra $\mathfrak{B}_{n}(x)$ was defined as the complex linear space with basis the set $\mathrm{Bd}_{n}$ of all Brauer $n$-diagrams, graphs on $2 n$ vertices and $n$ edges with the property that every vertex is incident to precisely one edge. The multiplication of two Brauer $n$-diagrams is defined using natural concatenation of diagrams. For more details, we refer the readers to [26] and [30]. Note that the subalgebra of $\mathfrak{B}_{n}(x)$ generated by $s_{1}, s_{2}, \cdots, s_{n-1}$ is isomorphic to the group algebra of the symmetric group $\mathfrak{S}_{n}$ over $\mathbb{Z}[x]$.

The Brauer algebra has been studied in a number of references, e.g., [3, [4, 5, [7, 10, [17, 22, 23, 24, 30, 31], 32, [34, [48. To set up a Schur-Weyl duality for orthogonal groups, we only need certain specialized Brauer algebras which we now recall. Let $\mathfrak{B}_{n}(m):=$ $\mathbb{Z} \otimes_{\mathbb{Z}[x]} \mathfrak{B}_{n}(x)$, where $\mathbb{Z}$ is regarded as $\mathbb{Z}[x]$-algebra by specifying $x$ to $m$. Let $\mathfrak{B}_{n}(m)_{K}:=$ $K \otimes_{\mathbb{Z}} \mathfrak{B}_{n}(m)$, where $K$ is regarded as $\mathbb{Z}$-algebra in the natural way. Then there is a right action of the specialized Brauer algebra $\mathfrak{B}_{n}(m)_{K}$ on the $n$-tensor space $V^{\otimes n}$ which commutes with the natural left action of $G O(V)$. We recall the definition of this action. Let $\delta_{i, j}$ denote the value of the usual Kronecker delta. For any integer $i$ with $1 \leq i \leq m$, we set $i^{\prime}=m+1-i$. We fix an ordered basis $\left\{v_{1}, v_{2}, \cdots, v_{m}\right\}$ of $V$ such that

$$
\left(v_{i}, v_{j}\right)=\delta_{i, j^{\prime}}, \quad \forall 1 \leq i, j \leq m .
$$


The right action of $\mathfrak{B}_{n}(m)$ on $V^{\otimes n}$ is defined on generators by

$$
\begin{gathered}
\left(v_{i_{1}} \otimes \cdots \otimes v_{i_{n}}\right) s_{j}:=v_{i_{1}} \otimes \cdots \otimes v_{i_{j-1}} \otimes v_{i_{j+1}} \otimes v_{i_{j}} \otimes v_{i_{j+2}} \otimes \cdots \otimes v_{i_{n}}, \\
\left(v_{i_{1}} \otimes \cdots \otimes v_{i_{n}}\right) e_{j}:=\delta_{i_{j}, i_{j+1}^{\prime}} v_{i_{1}} \otimes \cdots \otimes v_{i_{j-1}} \otimes\left(\sum_{k=1}^{m} v_{k} \otimes v_{k^{\prime}}\right) \otimes v_{i_{j+2}} \\
\otimes \cdots \otimes v_{i_{n}} .
\end{gathered}
$$

That is, the action of $s_{j}$ is by place permutation and the action of $e_{j}$ is by a composition of Weyl's "contraction" operator with an "expansion" operator. Let $\varphi$ be the $K$-algebra homomorphism

$$
\varphi:\left(\mathfrak{B}_{n}(m)\right)^{\text {op }} \rightarrow \operatorname{End}_{K}\left(V^{\otimes n}\right)
$$

induced by the above action.

Lemma 1.1. ([3], [4, [5]) 1) The natural left action of $G O(V)$ on $V^{\otimes n}$ commutes with the right action of $\mathfrak{B}_{n}(m)$. Moreover, if $K=\mathbb{C}$, then

$$
\begin{aligned}
& \varphi\left(\mathfrak{B}_{n}(m)_{\mathbb{C}}^{\text {op }}\right)=\operatorname{End}_{\mathbb{C} G O(V)}\left(V^{\otimes n}\right)=\operatorname{End}_{\mathbb{C} O(V)}\left(V^{\otimes n}\right), \\
& \psi(\mathbb{C} G O(V))=\psi(\mathbb{C} O(V))=\operatorname{End}_{\mathfrak{B}_{n}(m)_{\mathbb{C}}}\left(V^{\otimes n}\right),
\end{aligned}
$$

2) if $K=\mathbb{C}$ and $m \geq n$ then $\varphi$ is injective, and hence an isomorphism onto $\operatorname{End}_{\mathbb{C} G O(V)}\left(V^{\otimes n}\right)$,

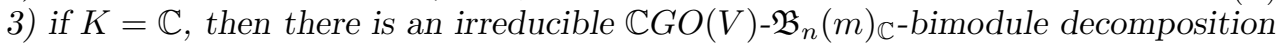

$$
V^{\otimes n}=\bigoplus_{f=0}^{[n / 2]} \bigoplus_{\substack{\lambda \vdash n-2 f \\ \lambda_{1}^{\prime}+\lambda_{2}^{\prime} \leq m}} \Delta(\lambda) \otimes D(\lambda),
$$

where $\Delta(\lambda)$ (respectively, $D(\lambda)$ ) denotes the irreducible $\mathbb{C} G O(V)$-module (respectively, the irreducible $\mathfrak{B}_{n}(m)$-module) corresponding to $\lambda$, and $\lambda^{\prime}=\left(\lambda_{1}^{\prime}, \lambda_{2}^{\prime}, \cdots\right)$ denotes the conjugate partition of $\lambda$.

The first main result in this work removes the restriction on $K$ in part 1) and part 2) of the above theorem. We have

THEOREM 1.2. For any infinite field $K$ of odd characteristic, we have

(a) $\psi(K G O(V))=\operatorname{End}_{\mathfrak{B}_{n}(m)}\left(V^{\otimes n}\right)$;

(b) $\varphi\left(\mathfrak{B}_{n}(m)\right)=\operatorname{End}_{K G O(V)}\left(V^{\otimes n}\right)=\operatorname{End}_{K O(V)}\left(V^{\otimes n}\right)$, and if $m \geq n$, then $\varphi$ is also injective, and hence an isomorphism onto

$$
\operatorname{End}_{K G O(V)}\left(V^{\otimes n}\right) .
$$

We remark that the first statement in part b) first appeared in [17] based on a completely different approach. The algebra

$$
S_{K}^{o}(m, n):=\operatorname{End}_{\mathfrak{B}_{n}(m)}\left(V^{\otimes n}\right)
$$

is called the orthogonal Schur algebra associated to $G O(V)$. Note that we use a different definition of orthogonal Schur algebra in Section 2 by defining $S_{K}^{o}(m, n)$ to be the linear dual of a certain coalgebra; the two definitions are reconciled in $(2.3)$. Let $\mathcal{R}=\mathbb{Z}[1 / 2]$. Let $V_{\mathcal{R}}$ be the free $\mathcal{R}$-module generated by $v_{1}, \cdots, v_{m}$. Let $\mathfrak{B}_{n}(m)_{\mathcal{R}}$ be the Brauer algebra defined over $\mathcal{R}$. We set

$$
S_{\mathcal{R}}^{o}(m, n):=\operatorname{End}_{\mathfrak{B}_{n}(m)_{\mathcal{R}}}\left(V_{\mathcal{R}}^{\otimes n}\right)
$$


In the course of our proof of Theorem 1.2, we show that the orthogonal Schur algebra is stable under base change, and the dimensions of both the orthogonal Schur algebra and the endomorphism algebra $\operatorname{End}_{K G O(V)}\left(V^{\otimes n}\right)$ are independent of the infinite field $K$ as long as char $K \neq 2$, see Corollary 2.7 and Lemma 3.7. We have also the following result:

THeOrem 1.3. Let $K$ be an infinite field of odd characteristic and let $O M_{m}(\bar{K})$ be the orthogonal monoid defined over the algebraic closure of $K$. Suppose $m$ is even. Then $O M_{m}(\bar{K})$ has two connected components, say $O M_{m}^{+}(\bar{K}), O M_{m}^{-}(\bar{K})$, where $O M_{m}^{+}(\bar{K})$ is the component containing the identity. Both components $O M_{m}^{+}(\bar{K}), O M_{m}^{-}(\bar{K})$ are normal varieties, and $\mathrm{OM}_{m}^{+}(\bar{K})$ is a reductive normal algebraic monoid. In particular, the orthogonal Schur algebra $S_{K}^{o,+}(m, n)$ associated to the identity component of $G O(V)$ is always a generalized Schur algebra in the sense of [15], [16].

When $m$ is odd, the orthogonal Schur algebra $S_{K}^{o}(m, n)$ is in general not a generalized Schur algebra. Note that the recent papers [39, 40, also study Schur algebras related to orthogonal groups.

As a consequence of Schur-Weyl duality, we know that the annihilator of the tensor space $V^{\otimes n}$ in the Brauer algebra $\mathfrak{B}_{n}(m)$ is stable under base change as long as char $K \neq 2$. Our second main result in this paper gives a characteristic-free description of this annihilator.

THEOREM 1.4. Let $K$ be an infinite field of odd characteristic and consider the partition of $n$ given by $\left(m+1,1^{n-m-1}\right):=(m+1, \underbrace{1, \cdots, 1}_{n-m-1 \text { copies }})$. We have that

$$
\operatorname{Ker} \varphi=\mathcal{M}_{K}^{\left(m+1,1^{n-m-1}\right)}
$$

where $\mathcal{M}_{K}^{\left(m+1,1^{n-m-1}\right)}$ is the right $K\left[\mathfrak{S}_{2 n}\right]$-module associated to $\left(m+1,1^{n-m-1}\right)$ as defined in the paragraphs below Lemma 6.4. In particular, $\operatorname{Ker} \varphi$ has a Specht filtration, regarded as $K\left[\mathfrak{S}_{2 n}\right]$-module.

We refer the reader to Sections 6 and 7 for the definition of $\mathcal{M}_{K}^{\left(m+1,1^{n-m-1}\right)}$ and the action of $\mathfrak{S}_{2 n}$ on it.

The paper is organized as follows. In Section 2 we prove the surjectivity of $\psi$. The proof is based on Cliff's basis for the coordinate algebra of orthogonal groups and a generalized Faddeev-Reshetikhin-Takhtajan's construction. We show that if $m$ is even, then each connected component of the orthogonal monoid is a normal variety. This implies that the orthogonal Schur algebra associated to the identity component is a generalized Schur algebra. In Section 3 we develop a tilting module theory for the orthogonal group $O_{m}(\bar{K})$. The main result there is that the tensor product of two tilting modules over $O_{m}(\bar{K})$ is again a tilting module. As a result, we deduce that the dimension of the endomorphism algebra of tensor space $V^{\otimes n}$ as a module over $O_{m}(K)$ does not depend on $K$ (for $\operatorname{char} K \neq 2$ ). Based on the results in Section 3, the surjectivity of $\varphi$ in the case where $m \geq n$ is proved in Section 4 in the same manner as $\mathbf{1 0}$, Section 3]. In Section 5, we prove the surjectivity of $\varphi$ in the case where $m \leq n$ in a similar way as [10, Section 4]. In Section 6, we study a permutation action of the symmetric group $\mathfrak{S}_{2 n}$ on the Brauer algebra. We construct a new $\mathbb{Z}$-basis for the resulting right $\mathfrak{S}_{2 n}$-module, which yields an integral filtration of Brauer algebra by right $\mathfrak{S}_{2 n}$-modules. Using these results and the Schur-Weyl duality we have proved, we give in Section 7 an explicit and characteristic-free description of the annihilator of tensor space $V^{\otimes n}$ in the Brauer algebra $\mathfrak{B}_{n}(m)$. 


\section{Orthogonal monoid and orthogonal Schur algebra}

Let $R$ be a noetherian integral domain such that $2 \cdot 1_{R}$ is invertible in $R$. Let $x_{i, j}, 1 \leq i, j \leq$ $m$, be $m^{2}$ commuting indeterminates over $R$. Let $A_{R}(m)$ be the free commutative $R$-algebra (i.e., polynomial algebra) in these $x_{i, j}, 1 \leq i, j \leq m$. Let $I_{R}$ be the ideal of $A_{R}(m)$ generated by elements of the form

$$
\left\{\begin{array}{l}
\sum_{k=1}^{m} x_{k, i} x_{k^{\prime}, j}, \quad 1 \leq i \neq j^{\prime} \leq m \\
\sum_{k=1}^{m} x_{i, k} x_{j, k^{\prime}}, \quad 1 \leq i \neq j^{\prime} \leq m \\
\sum_{k=1}^{m}\left(x_{k, i} x_{k^{\prime}, i^{\prime}}-x_{j, k} x_{j^{\prime}, k^{\prime}}\right), \quad 1 \leq i, j \leq m .
\end{array}\right.
$$

The $R$-algebra $A_{R}(m) / I_{R}$ will be denoted by $A_{R}^{o}(m)$. Write $c_{i, j}$ for the canonical image $x_{i, j}+$ $I_{R}$ of $x_{i, j}$ in $A_{R}^{o}(m)(1 \leq i, j \leq m)$. Then in $A_{R}^{o}(m)$ we have the relations

$$
\left\{\begin{array}{l}
\sum_{k=1}^{m} c_{k, i} c_{k^{\prime}, j}=0, \quad 1 \leq i \neq j^{\prime} \leq m ; \\
\sum_{k=1}^{m} c_{i, k} c_{j, k^{\prime}}=0, \quad 1 \leq i \neq j^{\prime} \leq m ; \\
\sum_{k=1}^{m}\left(c_{k, i} c_{k^{\prime}, i^{\prime}}-c_{j, k} c_{j^{\prime}, k^{\prime}}\right)=0, \quad 1 \leq i, j \leq m .
\end{array}\right.
$$

Note that $A_{R}(m)$ is a graded algebra, $A_{R}(m)=\oplus_{n>0} A_{R}(m, n)$, where $A_{R}(m, n)$ is the subspace spanned by the monomials of the form $x_{\underline{i}, \underline{j}}$ for $(\underline{i}, \underline{j}) \in I^{2}(m, n)$, where

$$
\begin{aligned}
& I(m, n):=\left\{\underline{i}=\left(i_{1}, \cdots, i_{n}\right) \mid 1 \leq i_{j} \leq m, \forall j\right\}, \\
& I^{2}(m, n)=I(m, n) \times I(m, n), \quad x_{\underline{i}, \underline{j}}:=x_{i_{1}, j_{1}} \cdots x_{i_{n}, j_{n}} .
\end{aligned}
$$

Since $I_{R}$ is a homogeneous ideal, $A_{R}^{o}(m)$ is graded too and

$$
A_{R}^{o}(m)=\oplus_{n \geq 0} A_{R}^{o}(m, n)
$$

where $A_{R}^{o}(m, n)$ is the subspace spanned by the monomials of the form $c_{\underline{i}, \underline{j}}$ for $(\underline{i}, \underline{j}) \in I^{2}(m, n)$, where

$$
c_{\underline{i}, \underline{j}}:=c_{i_{1}, j_{1}} \cdots c_{i_{n}, j_{n}} .
$$

By convention, throughout this paper, we identify the symmetric group $\mathfrak{S}_{n}$ with the set of maps acting on their arguments on the right. In other words, if $\sigma \in \mathfrak{S}_{n}$ and $a \in\{1, \ldots, n\}$ we write $(a) \sigma$ for the value of $a$ under $\sigma$. This convention carries the consequence that, when considering the composition of two symmetric group elements, the leftmost map is the first to act on its argument. For example, we have $(1,2,3)(2,3)=(1,3)$ in the usual cycle notation.

If one defines

$$
\Delta\left(x_{\underline{i}, \underline{j}}\right)=\sum_{\underline{k} \in I(m, n)} x_{\underline{i}, \underline{k}} \otimes x_{\underline{k}, \underline{j}}, \quad \varepsilon\left(x_{\underline{i}, \underline{j}}\right)=\delta_{\underline{i}, \underline{j}}, \forall \underline{i}, \underline{j} \in I(m, n), \forall n,
$$

then the algebra $A_{R}(m)$ becomes a graded bialgebra, and each $A_{R}(m, n)$ is a sub-coalgebra of $A_{R}(m)$. Its linear dual

$$
S_{R}(m, n):=\operatorname{Hom}_{R}\left(A_{R}(m, n), R\right)
$$


is the usual Schur algebra over $R$ (see [27]). Let

$$
S_{R}^{o}(m, n):=\operatorname{Hom}_{R}\left(A_{R}^{o}(m, n), R\right)
$$

the orthogonal Schur algebra. It is clear that $A_{R}^{o}(m, n)$ is in fact a quotient coalgebra of $A_{R}(m, n)$, hence $S_{R}^{o}(m, n)$ is a subalgebra of $S_{R}(m, n)$.

For any integers $i, j \in\{1,2, \cdots, m\}$, we let $E_{i, j}$ denote the corresponding matrix unit for $\operatorname{End}_{R}\left(V_{R}\right)$, where $V_{R}$ is a free $R$-module of rank $n$. We define

$$
\beta:=\sum_{1 \leq i, j \leq m} E_{i, j} \otimes E_{j, i}, \quad \gamma:=\sum_{1 \leq i, j \leq m} E_{i, j} \otimes E_{i^{\prime}, j^{\prime}}
$$

For $i=1,2, \cdots, n-1$, we set

$$
\beta_{i}:=\operatorname{id}_{V \otimes i-1} \otimes \beta \otimes \operatorname{id}_{V \otimes n-i-1}, \quad \gamma_{i}:=\operatorname{id}_{V \otimes i-1} \otimes \gamma \otimes \operatorname{id}_{V \otimes n-i-1} .
$$

By direct verification, it is easy to see that the map which sends $s_{i}$ to $\beta_{i}$ and $e_{i}$ to $\gamma_{i}$ for each $1 \leq i \leq n-1$ extends to a representation of $\mathfrak{B}_{n}(m)$ on $V_{R}^{\otimes n}$ which is nothing but the representation we have defined above Lemma 1.1. By [45, Theorem 3.3] and the discussion in [45, Section 5], we know that

$$
\operatorname{End}_{\mathfrak{B}_{n}(m)_{R}}\left(V_{R}^{\otimes n}\right) \cong S_{R}^{o}(m, n):=\operatorname{Hom}_{R}\left(A_{R}^{o}(m, n), R\right) .
$$

Let $i$ be an integer with $1 \leq i \leq m$. We define

$$
\operatorname{det}_{0}=\sum_{k=1}^{m} c_{k, i} c_{k^{\prime}, i^{\prime}} \in A_{R}^{o}(m, 2) .
$$

By the relations in (2.2), we know that $\operatorname{det}_{0}$ does not depend on the choice of $i$. It is well known (and easy to check) that $\operatorname{det}_{0}$ is a group-like element in the bialgebra $A_{R}^{o}(m)$. Note that the relations in (2.1) are equivalent to

$$
C^{t} J C=\operatorname{det}_{0} J, \quad C J C^{t}=\operatorname{det}_{0} J,
$$

where

$$
C:=\left(c_{i, j}\right)_{n \times n}, \quad J=\left(\begin{array}{ccccc}
0 & 0 & \cdots & 0 & 1 \\
0 & 0 & \cdots & 1 & 0 \\
\vdots & \vdots & \vdots & \vdots & \vdots \\
0 & 1 & \cdots & 0 & 0 \\
1 & 0 & \cdots & 0 & 0
\end{array}\right)_{n \times n}
$$

It follows that $\left(\operatorname{det}_{0}\right)^{n}=\operatorname{det}^{2}$, where

$$
\operatorname{det}:=\sum_{k=1}^{m}(-1)^{\ell(w)} c_{1, w(1)} c_{2, w(2)} \cdots c_{n, w(n)}
$$

denotes the usual determinant function.

Let $\bar{K}$ be the algebraic closure of $K$. Let $M_{m}(\bar{K})$ be the set of all $m \times m$ matrices over $\bar{K}$. Then $M_{m}(\bar{K})$ is a linear algebraic monoid over $\bar{K}$. We define the orthogonal monoid $O M_{m}(\bar{K})$ as follows:

$$
O M_{m}(\bar{K}):=\left\{\begin{array}{l|l}
A \in M_{m}(\bar{K}) \mid \begin{array}{c}
\text { there exists } d \in \bar{K}, \text { such that } \\
A^{t} J A=A J A^{t}=d J .
\end{array}
\end{array}\right\} .
$$

The coordinate algebra $\bar{K}\left[M_{m}\right]$ of $M_{m}(\bar{K})$ is isomorphic to $A_{\bar{K}}(m):=A_{K}(m) \otimes_{K}$ $\bar{K}$. The coordinate algebra of the general linear group $G L_{m}(\bar{K})$ is isomorphic to $\bar{K}\left[x_{i, j}, \operatorname{det}\left(x_{i, j}\right)_{m \times m}^{-1}\right]_{1 \leq i, j \leq m}$. The orthogonal similitude group $G O_{m}(\bar{K})$ is defined as

$$
G O_{m}(\bar{K}):=\left\{\begin{array}{l|l}
A \in G L_{m}(\bar{K}) & \begin{array}{c}
\text { there exists } d \in \bar{K}^{\times}, \text {such that } \\
A^{t} J A=A J A^{t}=d J
\end{array}
\end{array}\right\} .
$$


The natural embedding $\iota_{1}: G O_{m}(\bar{K}) \hookrightarrow G L_{m}(\bar{K})$ induces a surjective map $\iota_{1}^{\#}: \bar{K}\left[G L_{m}\right] \rightarrow$ $\bar{K}\left[G O_{m}\right]$. Similarly, the natural embedding $\iota_{0}: O M_{m}(\bar{K}) \hookrightarrow M_{m}(\bar{K})$ induces a surjective map $\iota_{0}^{\#}: \bar{K}\left[M_{m}\right] \rightarrow \bar{K}\left[O M_{m}\right]$. We use $\iota_{2}, \iota_{3}$ to denote the natural inclusion from $G O_{m}(\bar{K})$ into $O M_{m}(\bar{K})$ and the natural inclusion from $G L_{m}(\bar{K})$ into $M_{m}(\bar{K})$ respectively. Note that $G L_{m}(\bar{K})$ is a dense open subset of $M_{m}(\bar{K})$, and by [18, $\left.(6.6(\mathrm{e})),(7.6(\mathrm{~g}))\right], G O_{m}(\bar{K})$ is a dense open subset of $O M_{m}(\bar{K})$. Therefore, $\iota_{2}\left(\right.$ resp., $\left.\iota_{3}\right)$ induces an inclusion $\iota_{2}^{\#}$ from $\bar{K}\left[O M_{m}\right]$ into $\bar{K}\left[G O_{m}\right]$ (resp., an inclusion $\iota_{3}^{\#}$ from $\bar{K}\left[M_{m}\right]$ into $\bar{K}\left[G L_{m}\right]$. We denote by $\widetilde{A}_{\bar{K}}^{o}(m)$ (resp., $\widetilde{A}_{\bar{K}}^{o}(m, n)$ ) the image of $A_{\bar{K}}(m)$ (resp., of $A_{\bar{K}}(m, n)$ ) in $\bar{K}\left[G O_{m}\right]$. We define $\widetilde{A}_{K}^{o}(m)$ (resp., $\left.\widetilde{A}_{K}^{o}(m, n)\right)$ to be the image of $A_{K}(m)$ (resp., of $A_{K}(m, n)$ ) under the surjective map $\bar{K}\left[G L_{m}\right] \rightarrow \bar{K}\left[G O_{m}\right]$.

LEMma 2.4. With the notations as above, the algebra $\widetilde{A} \frac{o}{K}(m)$ is isomorphic to the coordinate algebra of the orthogonal monoid $O M_{m}(\bar{K})$. Moreover, the $\bar{K}$-dimension of $\widetilde{A}_{\bar{K}}^{o}(m, n)$ does not depend on the choice of the infinite field $K$ as long as char $K \neq 2$.

Proof. We have the following commutative diagram of maps:

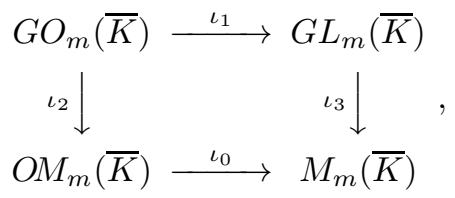

which induces the following commutative diagram:

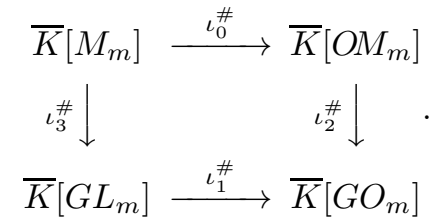

Since $\iota_{0}^{\#}$ is a surjection, while $\iota_{2}^{\#}$ is an injection, the first conclusion of the lemma follows immediately from the above commutative diagram.

It remains to prove the second conclusion. Let $\mathcal{R}:=\mathbb{Z}[1 / 2]$. In [6 Section 8], Cliff proved that for any field $K$ which is an $\mathcal{R}$-algebra, the elements in the following set

$$
\left\{\begin{array}{l|l}
\left(\operatorname{det}_{0}\right)^{k}[S: T] & \begin{array}{c}
k \in \mathbb{Z}, 0 \leq k \leq n / 2,[S: T] \text { is } O(m) \\
\text { standard of shape } \lambda, \lambda \vdash n-2 k
\end{array}
\end{array}\right\}
$$

forms a $K$-linear spanning set of $A_{K}^{o}(m, n)$.

By definition of $O M_{m}(K)$, it is easy to check that the defining relations (2.1) vanish on every matrix in $O M_{m}(K)$. It follows that there is a natural epimorphism of graded bialgebras from $A_{K}^{o}(m)$ onto $\widetilde{A}_{K}^{o}(m)$. Therefore, the image in $\widetilde{A}_{K}^{o}(m)$ of the elements in (2.5) for all $n \geq 0$ also form a $K$-linear spanning set of $\widetilde{A}_{K}^{o}(m)$. On the other hand, since the coordinate algebra of $G O_{m}(\bar{K})$ is just the localization of $O M_{m}(\bar{K})$ at $\operatorname{det}_{0}$, it follows that the image in $\bar{K}\left[G O_{m}\right]$ of the elements in the following set

$$
\left\{\begin{array}{l|l}
\left(\operatorname{det}_{0}\right)^{k}[S: T] & \begin{array}{c}
k \in \mathbb{Z},[S: T] \text { is } O(m) \text { standard } \\
\text { of shape } \lambda, \lambda \vdash r \in \mathbb{Z} \geq 0
\end{array}
\end{array}\right\}
$$

form a $\bar{K}$-linear spanning set of $\bar{K}\left[G O_{m}\right]$. If $K=\mathbb{C}$, Cliff proved that (in [6, Corollary 6.2]) the natural image of the elements in (2.5) for all $n \geq 0$ is actually a basis of $\mathbb{C}\left[O M_{m}\right]$, from which we deduce that the natural image of the elements in (2.6) are linearly independent in $\mathbb{C}\left[G O_{m}\right]$. 
By [9. Chapter II, $\S 5,(2.7)]$, we can deduce that the algebra $\bar{K}\left[G O_{m}\right]$ has a nice $\mathcal{R}$-form $\mathcal{R}\left[G O_{m}\right]$ such that the natural map

$$
\mathcal{R}\left[G O_{m}\right] \otimes_{\mathcal{R}} \bar{K} \rightarrow \bar{K}\left[G O_{m}\right]
$$

is an isomorphism. It follows that the elements in (2.6) are always linearly independent in $\bar{K}\left[G O_{m}\right]$. Since $\bar{K}\left[O M_{m}\right]$ is a subset of $\bar{K}\left[G O_{m}\right]$, we conclude that the image of the elements (2.5) in $\widetilde{A} \frac{o}{K}(m)$ also form a $K$-basis of $\bar{K}\left[O M_{m}\right]=\widetilde{A} \frac{o}{K}(m)$. In particular, $\operatorname{dim}_{K} A_{K}^{o}(m, n)=$ $\operatorname{dim}_{\bar{K}} \widetilde{A}_{\bar{K}}^{o}(m, n)$ is independent of the choice of the field $K$ as long as char $K \neq 2$. This completes the proof of the lemma.

By Lemma 2.4. for each $0 \leq n \in \mathbb{Z}$, the dimension of $\widetilde{A}_{K}^{o}(m, n)$ is independent of the field $K$. By [18, (9.5)], $A_{\mathbb{C}}^{o}(m, n) \cong \widetilde{A}_{\mathbb{C}}^{o}(m, n)$. By $(2.5), A_{K}^{o}(m, n)$ has a spanning set which has the same cardinality as $\operatorname{dim} \widetilde{A}_{\mathbb{C}}^{o}(m, n)$. Since $A_{K}^{o}(m, n)$ maps surjectively onto $\widetilde{A}_{K}^{o}(m, n)$, and by Lemma 2.4,

$$
\operatorname{dim} \widetilde{A}_{K}^{o}(m, n)=\operatorname{dim} \widetilde{A}_{\mathbb{C}}^{o}(m, n) .
$$

It follows that the elements in the spanning set (2.5) form an integral basis of $A_{K}^{o}(m, n)$, and thus the surjection from $A_{K}^{o}(m, n)$ to $\widetilde{A}_{K}^{o}(m, n)$ is always an isomorphism. It follows that $A_{K}^{o}(m, n) \cong \widetilde{A}_{K}^{o}(m, n)$ and $A_{K}^{o}(m) \cong \widetilde{A}_{K}^{o}(m)$. In particular, we have $S_{K}^{o}(m, n) \cong \widetilde{S}_{K}^{o}(m, n):=$ $\operatorname{Hom}_{K}\left(\widetilde{A}_{K}^{o}(m, n), K\right)$. Applying (2.3) , we get that

COROLlary 2.7. With the notation as above, we have that

1) $A_{R}^{o}(m, n)$ is a free $R$-module of finite rank, and for any commutative $\mathcal{R}$-algebra $K$, the natural map

$$
A_{\mathcal{R}}^{o}(m, n) \otimes_{\mathcal{R}} K \rightarrow A_{K}^{o}(m, n)
$$

is always an isomorphism.

2) $\operatorname{End}_{\mathfrak{B}_{n}(m)_{R}}\left(V_{R}^{\otimes n}\right)$ is a free $R$-module of finite rank, and for any commutative $\mathcal{R}$-algebra $K$, the natural map

$$
\operatorname{End}_{\mathfrak{B}_{n}(m)_{\mathcal{R}}}\left(V_{\mathcal{R}}^{\otimes n}\right) \otimes_{\mathcal{R}} K \rightarrow \operatorname{End}_{\mathfrak{B}_{n}(m)_{K}}\left(V_{K}^{\otimes n}\right)
$$

is always an isomorphism.

By [18, (4.4)], $G O_{m}(K)$ admits a graded polynomial representation theory in the sense of [18, (1.2)]. Applying [18, (3.2)], we deduce that the images of $K G O(V)$ and of $\widetilde{S}_{K}^{o}(m, n)$ in $\operatorname{End}\left(V_{K}^{\otimes n}\right)$ are the same. On the other hand, the natural isomorphisms $\widetilde{S}_{K}^{o}(m, n) \cong S_{K}^{o}(m, n) \cong$ $\operatorname{End}_{\mathfrak{B}_{n}(m)}\left(V^{\otimes n}\right)$ imply that the image of $\widetilde{S}_{K}^{o}(m, n)$ in $\operatorname{End}\left(V^{\otimes n}\right)$ is exactly $\operatorname{End}_{\mathfrak{B}_{n}(m)}\left(V^{\otimes n}\right)$. Therefore, we deduce that

$$
\psi(K G O(V))=\operatorname{End}_{\mathfrak{B}_{n}(m)}\left(V^{\otimes n}\right) .
$$

This completes the proof of part a) in Theorem 1.2 It also shows the isomorphism

$$
S_{K}^{o}(m, n) \cong \operatorname{End}_{\mathfrak{B}_{n}(m)}\left(V^{\otimes n}\right) ;
$$

so we see that the orthogonal Schur algebra may be regarded as an endomorphism algebra for the Brauer algebra.

From now on until the end of this section, we consider only the case where $m=2 l$. In [6. Section 8], Cliff proved that $G O_{2 l}(\bar{K})$ is isomorphic to $O_{2 l}(\bar{K}) \times \bar{K}^{\times}$as a variety. The 
isomorphism is given by

$$
\begin{aligned}
& \rho_{1}: \quad G O_{2 l}(\bar{K}) \rightarrow O_{2 l}(\bar{K}) \times \bar{K}^{\times} \\
& A \mapsto\left(A \xi_{1}\left(\operatorname{det}_{0} A\right)^{-1}, \operatorname{det}_{0} A\right),
\end{aligned}
$$

where $\xi_{1}(t):=\operatorname{diag}(\underbrace{t, \cdots, t}_{l \text { copies }}, \underbrace{1, \cdots, 1}_{l \text { copies }}), \forall t \in \bar{K}$. The inverse of $\rho_{1}$ is given by

$$
\begin{gathered}
\rho_{1}^{-1}: \quad O_{2 l}(\bar{K}) \times \bar{K}^{\times} \rightarrow G O_{2 l}(\bar{K}) \\
(A, c) \mapsto A \xi_{1}(c) .
\end{gathered}
$$

In this case, we have that $\bar{K}\left[G O_{2 l}\right] \cong \bar{K}\left[O_{2 l}\right] \otimes \bar{K}\left[T, T^{-1}\right]$. It also follows that $G O_{2 l}(\bar{K})$ has two connected components in this case.

As a regular function on $G O_{2 l}(\bar{K}), \operatorname{det}^{2}=\operatorname{det}_{0}^{2 l}$. Note that det $/ \operatorname{det}_{0}$ is also a regular function on $G O_{2 l}(\bar{K})$. It follows that the two connected components of $G O_{2 l}(\bar{K})$ must be

$$
\begin{aligned}
& G O_{2 l}^{+}(\bar{K}):=\left\{A \in G O_{2 l}(\bar{K}) \mid \operatorname{det} A=\left(\operatorname{det}_{0} A\right)^{l}\right\}, \\
& G O_{2 l}^{-}(\bar{K}):=\left\{A \in G O_{2 l}(\bar{K}) \mid \operatorname{det} A=-\left(\operatorname{det}_{0} A\right)^{l}\right\} .
\end{aligned}
$$

Note that in this case, $\operatorname{det} J=-1, J^{2}=I_{2 l \times 2 l}, J \in G O_{2 l}^{-}(\bar{K})$, and we have $G O_{2 l}^{-}(\bar{K})=$ $G O_{2 l}^{+}(\bar{K}) \cdot J=J \cdot G O_{2 l}^{+}(\bar{K})$. It is easy to check that $G O_{2 l}^{+}(\bar{K})$ is a (connected) reductive algebraic group. Let

$$
O_{2 l}^{+}(\bar{K}):=S O_{2 l}(\bar{K}), O_{2 l}^{-}(\bar{K}):=J \cdot S O_{2 l}(\bar{K}) .
$$

It is clear from the isomorphism $\rho_{1}$ that

$$
G O_{2 l}^{+}(\bar{K}) \cong O_{2 l}^{+}(\bar{K}) \times K^{\times}, G O_{2 l}^{-}(\bar{K}) \cong O_{2 l}^{-}(\bar{K}) \times K^{\times} .
$$

Let

$$
\begin{aligned}
& O M_{2 l}^{+}(\bar{K}):=\left\{A \in O M_{2 l}(\bar{K}) \mid \operatorname{det} A=\left(\operatorname{det}_{0} A\right)^{l}\right\}, \\
& O M_{2 l}^{-}(\bar{K}):=\left\{A \in O M_{2 l}(\bar{K}) \mid \operatorname{det} A=-\left(\operatorname{det}_{0} A\right)^{l}\right\} .
\end{aligned}
$$

Since $\overline{G O_{2 l}^{+}(\bar{K})} \subseteq O M_{2 l}^{+}(\bar{K}), \overline{G O_{2 l}^{-}(\bar{K})} \subseteq O M_{2 l}^{-}(\bar{K})$, and $G O_{2 l}(\bar{K})$ is a dense open set in $O M_{2 l}(\bar{K})$, it follows that $G O_{2 l}^{+}(\bar{K})$ (resp., $G O_{2 l}^{-}(\bar{K})$ ) is a dense open subset in $O M_{2 l}^{+}(\bar{K})$ (resp., in $O M_{2 l}^{-}(\bar{K})$ ). It follows that $O M_{2 l}^{+}(\bar{K}), O M_{2 l}^{-}(\bar{K})$ are the only two connected components $O M_{2 l}(\bar{K})$, and $I_{2 l \times 2 l} \in O M_{2 l}^{+}(\bar{K})$.

Theorem 2.9. Let $x$ be an indeterminant over $\bar{K}$. Then there is an embedding $\bar{K}\left[O M_{2 l}^{+}\right] \hookrightarrow$ $\bar{K}\left[O_{2 l}^{+}\right] \otimes \bar{K}[x]$, and we have the following commutative diagram

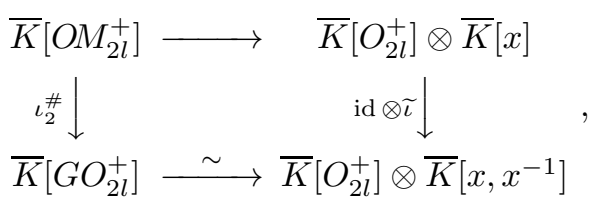

where the top horizontal map is the given embedding, $\widetilde{\iota}$ is the natural embedding $\bar{K}[x] \hookrightarrow$ $\bar{K}\left[x, x^{-1}\right]$. The same is true if we replace "+" by "-".

Proof. Let $f \in \bar{K}\left[O_{2 l}^{+}\right] \otimes \bar{K}\left[x, x^{-1}\right]$. We can write

$$
f=\sum_{i \in \mathbb{Z}} f_{i} \otimes x^{i},
$$


where $f_{i} \in \bar{K}\left[O_{2 l}^{+}\right]$for each $i$ and $\operatorname{supp} f:=\left\{i \in \mathbb{Z} \mid f_{i} \neq 0\right\}$ is a finite set. From (2.8) we have an isomorphism $\bar{K}\left[G O_{2 l}^{+}\right] \cong \bar{K}\left[O_{2 l}^{+}\right] \otimes \bar{K}\left[x, x^{-1}\right]$. One can show that the function $x^{i}$ on $G O_{2 l}^{+}$is given by $\operatorname{det}_{0}^{i}$. We identify $\bar{K}\left[O M_{2 l}^{+}\right]$with its image in $\bar{K}\left[G O_{2 l}^{+}\right]$, and $\bar{K}\left[G O_{2 l}^{+}\right]$with $\bar{K}\left[O_{2 l}^{+}\right] \otimes$ $\bar{K}\left[x, x^{-1}\right]$. We regard $O M_{2 l}^{+}(\bar{K})$ as a closed subvariety of $(\bar{K})^{4 l^{2}}$. Suppose that $f \in \bar{K}\left[O M_{2 l}^{+}\right]$. This means that $f$ can be extended to a regular function $\widetilde{f}$ on $O M_{2 l}^{+}(\bar{K})$. For each element $A \in$ $O M_{2 l}^{+}(\bar{K}) \backslash G O_{2 l}^{+}(\bar{K})$, we know (by definition) that $\operatorname{det}_{0} A=0$. Since $\widetilde{f} \in \bar{K}\left[O M_{2 l}^{+}\right]$, there must exist an open neighborhood $V_{A}$ of $A$ and two polynomials $g_{A}, h_{A} \in \bar{K}\left[x_{1,1}, x_{1,2}, \cdots, x_{2 l, 2 l}\right]$, such that for any $X \in V_{A}, h_{A}(X) \neq 0$ and $f(X)=g_{A}(X) / h_{A}(X)$. Note that the open subsets

$$
G O_{2 l}^{+}(\bar{K}), \quad V_{A}, A \in O M_{2 l}^{+}(\bar{K}) \backslash G O_{2 l}^{+}(\bar{K})
$$

gives a covering of $O M_{2 l}^{+}(\bar{K})$. Since an affine variety is a Noetherian topological space and $O M_{2 l}^{+}(\bar{K})$ is an infinite set, we can always find an element $A \in O M_{2 l}^{+}(\bar{K}) \backslash G O_{2 l}^{+}(\bar{K})$ such that

$$
V_{A} \bigcap\left(O M_{2 l}^{+}(\bar{K}) \backslash G O_{2 l}^{+}(\bar{K})\right)
$$

is an infinite set. We fix such an element $A$. We claim that $f_{i}=0$ whenever $i<0$. Suppose this is not the case. Let $i_{0}<0$ be the least integer such that $f_{i_{0}} \neq 0$. Then for any $X \in V_{A} \cap G O_{2 l}^{+}(\bar{K})$,

$$
h_{A}(X) f_{i_{0}}(X)+h_{A}(X)\left(\sum_{i_{0}<i \in \mathbb{Z}} f_{i}(X)\left(\operatorname{det}_{0} X\right)^{i-i_{0}}\right)-\left(\operatorname{det}_{0} X\right)^{-i_{0}} g_{A}(X)=0,
$$

Since $G O_{2 l}^{+}(\bar{K})$ is dense in $O M_{2 l}^{+}(\bar{K})$, it follows that $V_{A} \cap G O_{2 l}^{+}(\bar{K})$ contains infinitely many points. This means we have the following polynomial identity:

$$
h_{A} f_{i_{0}}+h_{A}\left(\sum_{i_{0}<i \in \mathbb{Z}} f_{i}\left(\operatorname{det}_{0}\right)^{i-i_{0}}\right)-\left(\operatorname{det}_{0}\right)^{-i_{0}} g_{A}=0 .
$$

On the other hand, since $V_{A} \bigcap\left(O M_{2 l}^{+}(\bar{K}) \backslash G O_{2 l}^{+}(\bar{K})\right)$ is an infinite set, we can always find a point $B \in V_{A} \bigcap\left(O M_{2 l}^{+}(\bar{K}) \backslash G O_{2 l}^{+}(\bar{K})\right)$ such that $f_{i_{0}}(B) \neq 0$. Now we evaluate the polynomial identity (2.10) at $B$ on both sides, we get a contradiction since two of the terms on the left hand side of (2.10) are zero (because $\operatorname{det}_{0}(B)=0$ ) and the other is nonzero. The contradiction proves our claim, and also completes the proof of the lemma.

Note that the map

$$
A \mapsto A J, \forall A \in O M_{2 l}^{-}(\bar{K}),
$$

defines a variety isomorphism $O M_{2 l}^{-}(\bar{K}) \cong O M_{2 l}^{+}(\bar{K})$. The following corollary proves the statements in Theorem 1.3.

Corollary 2.11. As a variety, $O M_{2 l}^{+}(\bar{K})$ is normal, and hence $O M_{2 l}^{+}(\bar{K})$ is a (connected) reductive normal algebraic monoid. In particular, in this case, $\mathrm{GO}_{2 l}^{+}$admits a polynomial representation theory in the sense of [18], and

$$
\widetilde{S}_{K}^{o,+}(2 l, n):=\operatorname{Hom}_{K}\left(\widetilde{A}_{K}^{o,+}(2 l, n), K\right)
$$

is a generalized Schur algebra in the sense of [15] and [16], where $\widetilde{A}_{K}^{o,+}(2 l, n)$ denotes the image of $A_{K}(2 l, n)$ in the coordinate algebra $\bar{K}\left[\mathrm{OM}_{2 l}^{+}\right]$.

Proof. Since $S O_{2 l}(\bar{K})=O_{2 l}^{+}(\bar{K})$ is an irreducible smooth affine variety, it follows that $\bar{K}\left[S O_{2 l}\right]$ is a normal domain. By [20, Exercise 4.18], $\bar{K}\left[S O_{2 l}\right] \otimes K[x]$ is a normal domain too. Let $A:=\bar{K}\left[O M_{2 l}^{+}\right], B:=\bar{K}\left[S O_{2 l}\right] \otimes K[x]$. Then the fraction field of $A$ is a subfield of the fraction field of $B$. Now let $z$ be an element in the fraction field of $A$, such that

$$
a_{0}+a_{1} z+\cdots+a_{k-1} z^{k-1}+z^{k}=0,
$$


for some $k \in \mathbb{N} \cup\{0\}$ and $a_{0}, a_{1}, \cdots, a_{k-1} \in A$. Since $B$ is normal, it follows that $z \in B$. On the other hand, note that det $=\operatorname{det}_{0}^{l}$ as a regular function on $O M_{2 l}^{+}(\bar{K})$, by the definition of the embedding $A \hookrightarrow B$. From this it is easy to see that a polynomial $f=\sum_{i \in \mathbb{Z}} f_{i} \otimes x^{i} \in B \backslash A$ if and only if

$$
f=g / \operatorname{det}_{0}^{k},
$$

for some $k \in \mathbb{Z}^{\geq 0}, g \in K\left[x_{1,1}, x_{1,2}, \cdots, x_{2 l, 2 l}\right]$ satisfying $\operatorname{det}_{0} \nmid g$. It follows that $B \backslash A$ is closed under multiplication. Applying [2, Chapter 5, Exercise 7], we deduce that $x \in A$. This proves that $A$ is normal. Hence $O M_{2 l}^{+}(\bar{K})$ is normal. Since its group of units $G O_{2 l}^{+}(\bar{K})$ is a reductive group, it follows that $O M_{2 l}^{+}(\bar{K})$ is a reductive normal algebraic monoid. It is easy to check that $0 \in O M_{2 l}^{+}(\bar{K})$ and $O M_{2 l}^{+}(\bar{K})$ has one-dimensional center. Using [19, Theorem 4.4], we deduce that $G O_{2 l}^{+}$admits a polynomial representation theory in the sense of [18], and $\widetilde{S}_{K}^{o,+}(2 l, n):=$ $\operatorname{Hom}_{K}\left(\widetilde{A}_{K}^{o,+}(2 l, n), K\right)$ is a generalized Schur algebra in the sense of [15] and [16].

Finally, we remark that the same argument can be used to show that the symplectic monoid ([18, 45) $S p M_{2 l}(\bar{K})$ is also a connected reductive normal algebraic monoid.

\section{Tilting modules over orthogonal groups}

The purpose of this section is to develop a tilting module theory for orthogonal groups. Note that in the literature the theory of tilting modules was well established for connected reductive algebraic groups, and the existence of a tilting module theory for orthogonal groups was only announced in [1] without full details.

Let $K$ be an infinite field of odd characteristic, $\bar{K}$ be its algebraic closure. By restriction, $V$ becomes a module over the special orthogonal group $S O_{m}(\bar{K})$. In this case, $V \cong L\left(\varepsilon_{1}\right)=$ $\Delta\left(\varepsilon_{1}\right)=\nabla\left(\varepsilon_{1}\right)$ is a tilting module over $S O_{m}(\bar{K})$. By the general theory of tilting modules over semi-simple algebraic groups (cf. [33, Chapter E]), we know that $V_{\bar{K}}^{\otimes n}$ is also a tilting module over $S O_{m}(\bar{K})$, and the dimension of

$$
\operatorname{End}_{S O_{m}(\bar{K})}\left(V_{\bar{K}}^{\otimes n}\right)
$$

does not depend on the choice of the field $K$.

Let $\theta \in G L(V)$ which is defined on the basis $\left\{v_{i}\right\}_{1 \leq i \leq m}$ by

$$
\theta\left(v_{i}\right)=\left\{\begin{array}{ll}
v_{i^{\prime}}, & \text { if } i=m / 2 \text { or } i=m / 2+1 ; \\
v_{i}, & \text { otherwise. }
\end{array}, i=1,2, \cdots, m,\right.
$$

if $m$ is even; or

$$
\theta\left(v_{i}\right)=-v_{i}, \quad i=1,2, \cdots, m,
$$

if $m$ is odd. Note that $\theta$ is an order 2 element in $O_{m}(K)$, and $O_{m}(K)$ is generated by $S O_{m}(K)$ and $\theta$.

For the moment we assume that $m=2 l$ is even, and $K=\bar{K}$. Let $G:=O_{m}(K), H:=$ $S O_{m}(K)$. We set

$$
T:=\left\{\operatorname{diag}\left(t_{1}, \cdots, t_{l}, t_{l}^{-1}, \cdots, t_{1}^{-1}\right) \mid t_{1}, \cdots, t_{l} \in K^{\times}\right\} .
$$

Then $T$ is a closed subgroup of $H$. In fact, $T$ is a maximal torus of $H$. Clearly, $\theta T \theta^{-1}=T$. Let $W:=N_{H}(T) / T$ be the Weyl group of $H$. For each integer $i$ with $1 \leq i \leq m$, let $\varepsilon_{i}$ be the function which sends a diagonal matrix in $G L_{m}$ to its $i$ th element in the diagonal. We identify 
a weight $\lambda \varepsilon_{1}+\cdots+\lambda_{l} \varepsilon_{l} \in X(T)$ with the sequence $\lambda=\left(\lambda_{1}, \cdots, \lambda_{l}\right)$ of integers. Let $s_{0}$ be the generator of the cyclic group $\mathbb{Z} / 2 \mathbb{Z}$. There is a natural action of $\mathbb{Z} / 2 \mathbb{Z}$ on $X(T)$ which is defined on generators by:

$$
s_{0}(\lambda)=\left(\lambda_{1}, \lambda_{2}, \cdots,-\lambda_{l}\right), \quad \forall \lambda=\left(\lambda_{1}, \lambda_{2}, \cdots, \lambda_{l}\right) \in X(T) .
$$

For each $\lambda \in X(T)^{+}$(the set of dominant weights), we use $L(\lambda), \Delta(\lambda), \nabla(\lambda)$ to denote the corresponding simple module, Weyl module and co-Weyl module over $H$ respectively. If $s_{0}(\lambda)=$ $\lambda$, then we let $\theta$ act as id (resp., as - id) on the highest weight vector of $\Delta(\lambda)$. It is well known that (see [26. (5.2.2)]) this extends to a representation of $G$ on $\Delta(\lambda)$. The resulting $G$-module will be denoted by $\widetilde{\Delta}^{+}(\lambda)$ (resp., by $\widetilde{\Delta}^{-}(\lambda)$ ). In this case,

$$
\operatorname{Ind}_{H}^{G} \Delta(\lambda) \cong \widetilde{\Delta}^{+}(\lambda) \oplus \widetilde{\Delta}^{-}(\lambda) .
$$

If $s_{0}(\lambda) \neq \lambda$, then we set

$$
\widetilde{\Delta}^{0}(\lambda):=\operatorname{Ind}_{H}^{G} \Delta(\lambda), \quad \widetilde{\nabla}^{0}(\lambda):=\operatorname{Ind}_{H}^{G} \nabla(\lambda) .
$$

In a similar way, we can define $\widetilde{L}^{+}(\lambda), \widetilde{L}^{-}(\lambda)$ if $s_{0}(\lambda)=\lambda$; and $\widetilde{L}^{0}(\lambda)$ if $s_{0}(\lambda) \neq \lambda$. Using the fact that $\operatorname{Ind}_{H}^{G}$ is an exact functor and $\theta$ permutes the set of $H$-submodules of any $G$-module $M$, we deduce easily the next lemma.

Lemma 3.1. With the notations as above, the set

$$
\left\{\widetilde{L}^{+}(\lambda), \widetilde{L}^{-}(\lambda), \widetilde{L}^{0}(\mu) \mid \lambda, \mu \in X(T)^{+}, s_{0}(\lambda)=\lambda, s_{0}(\mu) \neq \mu\right\}
$$

forms a complete set of pairwise non-isomorphic simple $G$-modules.

If $s_{0}(\lambda)=\lambda$, then we define $\widetilde{\nabla}^{+}(\lambda), \widetilde{\nabla}^{-}(\lambda)$ to be the duals of $\widetilde{\Delta}^{+}\left(-w_{0} \lambda\right), \widetilde{\Delta}^{-}\left(-w_{0} \lambda\right)$ (where $w_{0}$ is the longest element in the Weyl group of $H$ ) such that $\theta$ also acts as id (resp., as - id) on the highest weight vector of $\widetilde{\nabla}^{+}(\lambda)$ (resp., of $\widetilde{\nabla}^{+}(\lambda)$ ). In this case it is also easy to show that

$$
\operatorname{Ind}_{H}^{G} \nabla(\lambda) \cong \widetilde{\nabla}^{+}(\lambda) \oplus \widetilde{\nabla}^{-}(\lambda) .
$$

We shall call $\widetilde{\Delta}^{+}(\lambda), \widetilde{\Delta}^{-}(\lambda), \widetilde{\Delta}^{0}(\lambda)$ the Weyl modules for $G$, and call $\widetilde{\nabla}^{+}(\lambda), \widetilde{\nabla}^{-}(\lambda), \widetilde{\nabla}^{0}(\lambda)$ the co-Weyl modules for $G$.

Lemma 3.2. Let $\lambda, \mu \in X(T)^{+}, x, y \in\{+,-, 0\}$, then

$$
\operatorname{Ext}_{G}^{i}\left(\widetilde{\Delta}^{x}(\lambda), \widetilde{\nabla}^{y}(\mu)\right)= \begin{cases}K, & \text { if } i=0, \lambda=\mu \text { and } x=y \\ 0, & \text { otherwise. }\end{cases}
$$

Proof. First, it is easy to see that if $x, y \in\{+,-\}$, then

$$
\operatorname{Hom}_{G}\left(\widetilde{\Delta}^{x}(\lambda), \widetilde{\nabla}^{y}(\mu)\right)= \begin{cases}K, & \text { if } \lambda=\mu \text { and } x=y ; \\ 0, & \text { otherwise. }\end{cases}
$$

Since $O_{m}$ is a flat group scheme (c.f. [6, 7.2]) over $\mathcal{R}$ and $S O_{m}$ is a normal subgroup scheme of $O_{m}$ (hence $S O_{m}$ is exact in $O_{m}$ ), we can apply [33, Part 1, Corollary (4.6)]. We divide the proof into two cases: 
Case 1. $s_{0}(\mu)=\mu, y \in\{+,-\}$. By [33, Part 1, Corollary (4.6)], we have

$$
\begin{aligned}
& \operatorname{Ext}_{G}^{i}\left(\widetilde{\Delta}^{x}(\lambda), \widetilde{\nabla}^{+}(\mu)\right) \oplus \operatorname{Ext}_{G}^{i}\left(\widetilde{\Delta}^{x}(\lambda), \widetilde{\nabla}^{-}(\mu)\right) \\
= & \operatorname{Ext}_{G}^{i}\left(\widetilde{\Delta}^{x}(\lambda), \widetilde{\nabla}^{+}(\mu) \oplus \widetilde{\nabla}^{-}(\mu)\right) \\
= & \operatorname{Ext}_{G}^{i}\left(\widetilde{\Delta}^{x}(\lambda), \operatorname{Ind}_{H}^{G} \nabla(\mu)\right) \\
\cong & \operatorname{Ext}_{H}^{i}\left(\operatorname{Res}_{H}^{G}\left(\widetilde{\Delta}^{x}(\lambda)\right), \nabla(\mu)\right) \\
= & \begin{cases}K, & \text { if } i=0, \lambda=\mu, x \in\{+,-\} \\
0, & \text { otherwise. }\end{cases}
\end{aligned}
$$

With (3.3), the above calculation shows that

$$
\operatorname{Ext}_{G}^{i}\left(\widetilde{\Delta}^{x}(\lambda), \widetilde{\nabla}^{y}(\mu)\right)= \begin{cases}K, & \text { if } i=0, \lambda=\mu \text { and } x=y \\ 0, & \text { otherwise }\end{cases}
$$

as required.

Case 2. $s_{0}(\mu) \neq \mu, y=0$. By [33, Part 1, Corollary (4.6)], we have

$$
\begin{aligned}
\operatorname{Ext}_{G}^{i}\left(\widetilde{\Delta}^{x}(\lambda), \widetilde{\nabla}^{0}(\mu)\right) & \cong \operatorname{Ext}_{G}^{i}\left(\widetilde{\Delta}^{x}(\lambda), \operatorname{Ind}_{H}^{G} \nabla(\mu)\right) \\
& \cong \operatorname{Ext}_{H}^{i}\left(\operatorname{Res}_{H}^{G}\left(\widetilde{\Delta}^{x}(\lambda)\right), \nabla(\mu)\right) \\
& = \begin{cases}K, & \text { if } i=0, \lambda=\mu, x=0 \\
0, & \text { otherwise },\end{cases}
\end{aligned}
$$

as required. This completes the proof of the lemma.

REMARK 3.4. Recall that $\mathcal{R}:=\mathbb{Z}[1 / 2]$. Let $H_{\mathcal{R}}$ be the $\mathcal{R}$-form of the special orthogonal group scheme $S O_{m}$. We define (cf. [33, Part I, (2.6)]) $G_{\mathcal{R}}:=H_{\mathcal{R}} \rtimes \mathbb{Z} / 2 \mathbb{Z}$. Let $\lambda \in X(T)^{+}$. By the representation theory of semi-simple algebraic groups (cf. [14 Lemma 11.5.3], [33, Part II, Chapter B]), we know that both the Weyl module $\Delta(\lambda)$ and the co-Weyl module $\nabla(\lambda)$ have nice $\mathcal{R}$-forms. We denote them by $\Delta_{\mathcal{R}}(\lambda), \nabla_{\mathcal{R}}(\lambda)$ respectively. Furthermore, for any $\mu \in X(T)^{+}, \nabla_{\mathcal{R}}(\lambda) \otimes \nabla_{\mathcal{R}}(\mu)$ has a $\nabla$-filtration, i.e., a filtration of $H_{\mathcal{R}}$-modules such that each successive quotient is isomorphic to some $\nabla_{\mathcal{R}}(\nu)$ for some $\nu \in X(T)^{+}$. The same is true for the $\Delta$-filtration of $\Delta_{\mathcal{R}}(\lambda) \otimes \Delta_{\mathcal{R}}(\mu)$. As a consequence, we can define the $R$-forms $\widetilde{\Delta}_{\mathcal{R}}^{x}(\lambda), \widetilde{\nabla}_{\mathcal{R}}^{x}(\lambda)$ $(x \in\{+,-, 0\})$ in a similar way, and Lemma 3.2 remains true if we replace everything by their $\mathcal{R}$-forms.

For any finite dimensional $G$-module $M$, an ascending filtration $0=M_{0} \subset M_{1} \subset \cdots \subset M$ of $G$-submodules is called a $\widetilde{\Delta}$-filtration (resp., $\widetilde{\nabla}$-filtration) if each successive quotient is isomorphic to some $\widetilde{\Delta}^{x}(\lambda)$ (resp., some $\widetilde{\nabla}^{x}(\lambda)$ ), where $\lambda \in X(T)^{+}, x \in\{+,-, 0\}$. A $G$-module is called a tilting module if it has both $\widetilde{\Delta}$-filtration and $\widetilde{\nabla}$-filtration.

Lemma 3.5. Let $\lambda, \mu \in X(T)^{+}, x, y \in\{+,-, 0\}$. The $G$-module $\widetilde{\Delta}^{x}(\lambda) \otimes \widetilde{\Delta}^{y}(\mu)$ has a $\Delta$ filtration and the $G$-module $\widetilde{\nabla}^{x}(\lambda) \otimes \widetilde{\nabla}^{y}(\mu)$ has a $\nabla$-filtration. In particular, the tensor product of any two tilting modules over $G$ is again a tilting module over $G$. 
Proof. Let $M:=\widetilde{\Delta}_{\mathcal{R}}^{x}(\lambda) \otimes \widetilde{\Delta}_{\mathcal{R}}^{y}(\mu)$. By the definition of $\widetilde{\Delta}_{\mathcal{R}}^{x}(\lambda)$ and $\widetilde{\Delta}_{\mathcal{R}}^{y}(\mu)$, it is easy to see that $\operatorname{Res}_{H}^{G} M$ has $\Delta$-filtration (as $H_{\mathcal{R}}$-module), say,

$$
0=M_{0} \subset M_{1} \subset M_{2} \subset \cdots \subset M_{s}=M .
$$

We set $N:=M_{1}$. Then $N \cong \Delta_{\mathcal{R}}(\nu)$ for some $\nu \in X(T)^{+}$. Let $\widetilde{N}:=N+\theta N$. Then $\widetilde{N}$ is a $G_{\mathcal{R}^{-}}$ submodule of $M$. It is clear that there is a surjective $G_{\mathcal{R}}$-homomorphism from $\operatorname{Ind}_{H_{\mathcal{R}}}^{G_{\mathcal{R}}}\left(\Delta_{\mathcal{R}}(\nu)\right)$ onto $\widetilde{N}$. We denote it by $\rho: \operatorname{Ind}_{H_{\mathcal{R}}}^{G_{\mathcal{R}}}\left(\Delta_{\mathcal{R}}(\nu)\right) \rightarrow \widetilde{N}$.

Note that both $\operatorname{Ind}_{H_{\mathcal{R}}}^{G_{\mathcal{R}}}\left(\Delta_{\mathcal{R}}(\nu)\right)$ and $\widetilde{N}$ are free $\mathcal{R}$-submodules of $M$. If $\operatorname{rank}_{\mathcal{R}} \operatorname{Ind}_{H_{\mathcal{R}}}^{G_{\mathcal{R}}}\left(\Delta_{\mathcal{R}}(\nu)\right)=$ $\operatorname{rank}_{\mathcal{R}} \tilde{N}$, then it is readily seen that $\rho$ is an isomorphism. That is, $\widetilde{N} \cong \operatorname{Ind}_{H_{\mathcal{R}}}^{G_{\mathcal{R}}}\left(\Delta_{\mathcal{R}}(\nu)\right)$ as $G$-module. Note that

$$
\operatorname{Ind}_{H_{\mathcal{R}}}^{G_{\mathcal{R}}}\left(\Delta_{\mathcal{R}}(\nu)\right)=\widetilde{\Delta}_{\mathcal{R}}^{0}(\nu)
$$

if $s_{0}(\nu) \neq \nu$; or

$$
\operatorname{Ind}_{H_{\mathcal{R}}}^{G_{\mathcal{R}}}\left(\Delta_{\mathcal{R}}(\nu)\right) \cong \widetilde{\Delta}_{\mathcal{R}}^{+}(\nu) \oplus \widetilde{\Delta}_{\mathcal{R}}^{-}(\nu),
$$

if $s_{0}(\nu)=\nu$. Clearly $\operatorname{Res}_{H_{\mathcal{R}}}^{G_{\mathcal{R}}}(\widetilde{N})$ has a $\Delta$-filtration. Applying [33, Part II, Lemma B.9, Corollary 4.17)], both $M$ and $M / \widetilde{N}$ have $\widetilde{\Delta}$-filtrations, so $M / \widetilde{N}$ also has a $\widetilde{\Delta}$-filtration. Now it follows easily by induction on $\operatorname{dim} M$ that $M$ has a $\widetilde{\Delta}$-filtration as $G$-module, as required.

Now we assume that

$$
\operatorname{rank}_{\mathcal{R}} \operatorname{Ind}_{H_{\mathcal{R}}}^{G_{\mathcal{R}}}\left(\Delta_{\mathcal{R}}(\nu)\right)>\operatorname{rank}_{\mathcal{R}} \widetilde{N}
$$

Let $\phi_{1}, \phi_{2}$ be the following two maps:

$$
\begin{array}{rlrl}
\phi_{1}: \quad \theta N \otimes_{\mathcal{R}} \mathbb{C} & \rightarrow \widetilde{N} \otimes_{\mathcal{R}} \mathbb{C} & \phi_{2}: \quad N \otimes_{\mathcal{R}} \mathbb{C} & \rightarrow \widetilde{N} \otimes_{\mathcal{R}} \mathbb{C} \\
\theta x \otimes_{\mathcal{R}} c & \mapsto \theta x \otimes_{\mathcal{R}} c & x \otimes_{\mathcal{R}} c & \mapsto x \otimes_{\mathcal{R}} c,
\end{array}
$$

where $x \in N, c \in \mathbb{C}$. Note that

$$
\theta N \otimes_{\mathcal{R}} \mathbb{C} \cong \Delta_{\mathbb{C}}\left(s_{0}(\nu)\right), \quad N \otimes_{\mathcal{R}} \mathbb{C} \cong \Delta_{\mathbb{C}}(\nu)
$$

are two simple $S O_{m}(\mathbb{C})$-modules, and

$$
\operatorname{Res}_{H_{\mathbb{C}}}^{G_{\mathbb{C}}}\left(\widetilde{N} \otimes_{\mathcal{R}} \mathbb{C}\right)=\operatorname{im}\left(\phi_{1}\right)+\operatorname{im}\left(\phi_{2}\right)
$$

Since $\theta^{2}=1$, it follows easily that $\phi_{1} \neq 0$ if and only if $\phi_{2} \neq 0$. Therefore, it follows from our assumption (3.6) that $s_{0}(\nu)=\nu$ and

$$
\operatorname{Res}_{H_{\mathbb{C}}}^{G_{\mathbb{C}}}\left(\widetilde{N} \otimes_{\mathcal{R}} \mathbb{C}\right)=\Delta_{\mathbb{C}}(\nu) .
$$

Therefore, we deduce that as $G_{\mathbb{C}}$-module, either

$$
\widetilde{N} \otimes_{\mathcal{R}} \mathbb{C} \cong \widetilde{\Delta}_{\mathbb{C}}^{+}(\nu),
$$

or

$$
\widetilde{N} \otimes_{\mathcal{R}} \mathbb{C} \cong \widetilde{\Delta}_{\mathbb{C}}^{-}(\nu) .
$$

In particular, $\theta$ always acts as a scalar on the highest weight vector of $\widetilde{N}$, and the scalar is either 1 or -1 . This implies that $N=\theta N$, and hence $\widetilde{N}=N+\theta N=N$, and either $\widetilde{N} \cong \widetilde{\Delta}_{\mathcal{R}}^{+}(\nu)$ or $\widetilde{N} \cong \widetilde{\Delta}_{\mathcal{R}}^{-}(\nu)$, as required. Now using the same argument as before, we can prove by induction that $M$ has a $\widetilde{\Delta}$-filtration as $G$-module. This proves the first statement of this lemma. Since every co-Weyl module is the dual of some Weyl module, the statement for $\widetilde{\nabla}$-filtrations follows immediately by taking duals. This completes the proof of the lemma. 
Lemma 3.7. Let $m$ be an arbitrary natural number, and $K$ be an arbitrary infinite field of odd characteristic. Then the dimension of

$$
\operatorname{End}_{O_{m}(K)}\left(V_{K}^{\otimes n}\right)
$$

does not depend on the choice of the infinite field $K$.

Proof. First, we note that

$$
\operatorname{End}_{O_{m}(K)}\left(V_{K}^{\otimes n}\right) \otimes_{K} \bar{K}=\operatorname{End}_{O_{m}(\bar{K})}\left(V_{\bar{K}}^{\otimes n}\right) .
$$

Therefore, to prove the lemma, we can assume without less of generality that $K=\bar{K}$.

If $m$ is odd, then $\theta$ acts as - id on the tensor space $V^{\otimes n}$. In that case, it is clear that

$$
\operatorname{dim} \operatorname{End}_{O_{m}(K)}\left(V_{K}^{\otimes n}\right)=\operatorname{dim} \operatorname{End}_{S O_{m}(K)}\left(V_{K}^{\otimes n}\right) .
$$

It follows that (cf. the discussion at the beginning of this section) the dimension of

$$
\operatorname{End}_{O_{m}(K)}\left(V_{K}^{\otimes n}\right)
$$

does not depend on the choice of the field $K$ in this case.

Now we assume that $m$ is even. We apply Lemmas 3.2 and 3.5. It follows by induction through the filtrations that the dimension of

$$
\operatorname{End}_{O_{m}(K)}\left(V_{K}^{\otimes n}\right)
$$

again does not depend on the choice of the field $K$ in this case. This completes the proof of the lemma.

\section{Proof of part b) in Theorem 1.2 in the case $m \geq n$}

The purpose of this section is to give a proof of part b) in Theorem 1.2 in the case where $m \geq n$. Throughout this section, we assume that $m \geq n$.

By Lemma 1.1 and Lemma 3.7, we know that

$$
\operatorname{dim} \operatorname{End}_{O_{m}(K)}\left(V_{K}^{\otimes n}\right)=\operatorname{dim} \mathfrak{B}_{n}(m) .
$$

Therefore, in order to prove part b) in Theorem 1.2 in the case $m \geq n$, it suffices to show that $\varphi$ is injective in that case. Without loss of generality, we can assume that $K=\bar{K}$ is algebraically closed.

Our strategy to prove the injectivity of $\varphi$ is similar to that used in [10, Section 3]. First, we make some conventions on the left and right place permutation actions. Throughout the rest of this paper, for any $\sigma, \tau \in \mathfrak{S}_{n}, a \in\{1,2, \cdots, n\}$, we set

$$
(a)(\sigma \tau)=((a) \sigma) \tau, \quad(\sigma \tau)(a)=\sigma(\tau(a)) .
$$

In particular, we have $\sigma(a)=(a) \sigma^{-1}$. Therefore, for any $\underline{i}=\left(i_{1}, i_{2}, \cdots, i_{n}\right) \in I(m, n), w \in \mathfrak{S}_{n}$, we have

$$
\underline{i} w=\left(i_{1}, i_{2}, \cdots, i_{n}\right) w=\left(i_{w(1)}, i_{w(2)}, \cdots, i_{w(n)}\right),
$$

which gives the so-called right place permutation action:

$$
v_{\underline{i}} w=\left(v_{i_{1}} \otimes \cdots \otimes v_{i_{n}}\right) w=v_{i_{w(1)}} \otimes \cdots \otimes v_{i_{w(n)}}=v_{\underline{i} w} .
$$


We make a further reduction. Let $\widehat{V}$ be the same $K$-vector space as $V$, endowed with a different non-degenerate symmetric bilinear form $(,)_{1}$ as follows:

$$
\left(v_{i}, v_{j}\right)_{1}:=\delta_{i, j}, \forall 1 \leq i, j \leq m .
$$

Then the orthogonal group relative to $(,)_{1}$ is defined to be

$$
O(\widehat{V}):=\left\{g \in G L(V) \mid(g v, g w)_{1}=(v, w)_{1}, \forall v, w \in \widehat{V}\right\} .
$$

We fix an element $c_{0} \in K$ such that $c_{0}^{2}=-1$. Then it is easy to see that the following map

$$
\phi: v_{i} \mapsto \begin{cases}\left(v_{i}+v_{i^{\prime}}\right) / \sqrt{2}, & \text { if } 1 \leq i \leq m / 2 ; \\ c_{0}\left(v_{i}-v_{i^{\prime}}\right) / \sqrt{2}, & \text { if }(m+1) / 2<i \leq m ; \forall 1 \leq i \leq m, \\ v_{i}, & \text { if } i=(m+1) / 2 .\end{cases}
$$

extends to an isomorphism from the orthogonal space $\widehat{V}$ onto the orthogonal space $V$. We extend $\phi$ diagonally to an isomorphism (still denoted by $\phi$ ) from $\widehat{V}^{\otimes n}$ onto $V^{\otimes n}$. Let $x \in O(\widehat{V}), f \in \operatorname{End}_{K}\left(\widehat{V}^{\otimes n}\right)$. It is easy to see that $x \in O(\widehat{V})$ if and only if $\phi x \phi^{-1} \in O(V)$, and $f \in \operatorname{End}_{O(\widehat{V})}\left(\widehat{V}^{\otimes n}\right)$ if and only if $\phi f \phi^{-1} \in \operatorname{End}_{O(V)}\left(V^{\otimes n}\right)$. In other words, the map $\widetilde{\phi}: f \mapsto \phi f \phi^{-1}$ defines an isomorphism from the endomorphism algebra $\operatorname{End}_{O(\widehat{V})}\left(\widehat{V}^{\otimes n}\right)$ onto the endomorphism algebra $\operatorname{End}_{O(V)}\left(V^{\otimes n}\right)$. Recall that we have a natural map $\varphi$ from $\mathfrak{B}_{n}(m)$ to $\operatorname{End}_{O(V)}\left(V^{\otimes n}\right)$. Using the isomorphism $\phi$, we get a $K$-algebra homomorphism $\widehat{\varphi}$ from $\mathfrak{B}_{n}(m)$ to $\operatorname{End}_{O(\widehat{V})}\left(\widehat{V}^{\otimes n}\right)$ as follows:

$$
X \mapsto \phi^{-1} \varphi(X) \phi, \forall X \in \mathfrak{B}_{n}(m) .
$$

By direct calculation, one can verify that for any $\underline{i}=\left(i_{1}, \cdots, i_{n}\right) \in I(m, n), j \in\{1,2, \cdots, n-$ $1\}$,

$$
\begin{gathered}
\left(v_{i_{1}} \otimes \cdots \otimes v_{i_{n}}\right) \widehat{\varphi}\left(s_{j}\right):=v_{i_{1}} \otimes \cdots \otimes v_{i_{j-1}} \otimes v_{i_{j+1}} \otimes v_{i_{j}} \otimes v_{i_{j+2}} \otimes \cdots \otimes v_{i_{n}}, \\
\left(v_{i_{1}} \otimes \cdots \otimes v_{i_{n}}\right) \widehat{\varphi}\left(e_{j}\right):=\delta_{i_{j}, i_{j+1}} v_{i_{1}} \otimes \cdots \otimes v_{i_{j-1}} \otimes\left(\sum_{k=1}^{m} v_{k} \otimes v_{k}\right) \otimes v_{i_{j+2}} \\
\otimes \cdots \otimes v_{i_{n}} .
\end{gathered}
$$

To prove $\varphi$ is injective, it suffices to prove that $\widehat{\varphi}$ is injective. This will be done in the rest of this section.

In [25], the Brauer algebra was shown to be cellular. Enyang gave in [21] an explicit combinatorial cellular basis for Brauer algebra. Enyang's basis is in some sense similar to the Murphy basis for type $A$ Hecke algebra. It is indexed by certain bitableaux. In the remaining part of this section we shall use Enyang's results from [21. We shall only use his basis for the specialized Brauer algebra $\mathfrak{B}_{n}(m)$. We first recall some notations and notions.

Let $n$ be a natural number. A bipartition of $n$ is a pair $\left(\lambda^{(1)}, \lambda^{(2)}\right)$ of partitions of numbers $n_{1}$ and $n_{2}$ with $n_{1}+n_{2}=n$. The notions of Young diagram, bitableaux, etc., carry over easily. For example, if $\lambda:=\left(\lambda^{(1)}, \lambda^{(2)}\right)$ is a bipartition of $n$, then a $\lambda$-bitableau $\mathfrak{t}$ is defined to be a bijective map from the Young diagram $[\lambda]$ to the set $\{1,2, \cdots, n\}$. Thus $\mathfrak{t}$ is a pair $\left(\mathfrak{t}^{(1)}, \mathfrak{t}^{(2)}\right)$ of tableaux, where $\mathfrak{t}^{(1)}$ is a $\lambda^{(1)}$-tableau and $\mathfrak{t}^{(2)}$ is a $\lambda^{(2)}$-tableau. A bitableau $\mathfrak{t}=\left(\mathfrak{t}^{(1)}, \mathfrak{t}^{(2)}\right)$ is called row standard if the numbers increase along rows in both $\mathfrak{t}^{(1)}$ and $\mathfrak{t}^{(2)}$. For each integer $f$ with $0 \leq f \leq[n / 2]$, we set $\nu=\nu_{f}:=\left(\left(2^{f}\right),(n-2 f)\right)$, where $\left(2^{f}\right):=(\underbrace{2,2, \cdots, 2}_{f \text { copies }})$ and $(n-2 f)$ are considered as partitions of $2 f$ and $n-2 f$ respectively. So $\nu$ is a bipartition of $n$. Let $\mathfrak{t}^{\nu}$ be the standard $\nu$-bitableau in which the numbers $1,2, \cdots, n$ appear in order along successive rows of the first component tableau, and then in order along successive rows of the second 
component tableau. We define

$$
\mathfrak{D}_{f}:=\left\{\begin{array}{l|l}
d \in \mathfrak{S}_{n} & \begin{array}{c}
\left(\mathfrak{t}^{(1)}, \mathfrak{t}^{(2)}\right)=\mathfrak{t}^{\nu} d \text { is row standard and the first } \\
\text { column of } \mathfrak{t}^{(1)} \text { is an increasing sequence } \\
\text { when read from top to bottom }
\end{array}
\end{array}\right\} .
$$

For each partition $\lambda$ of $n-2 f$, we denote by $\operatorname{Std}(\lambda)$ the set of all the standard $\lambda$-tableaux with entries in $\{2 f+1, \cdots, n\}$. The initial tableau $\mathfrak{t}^{\lambda}$ in this case has the numbers $2 f+1, \cdots, n$ in order along successive rows.

Lemma 4.1. ([21]) For each $\lambda \vdash n-2 f, \mathfrak{s}, \mathfrak{t} \in \operatorname{Std}(\lambda)$, let $m_{\mathfrak{s}, \mathfrak{t}}$ be the corresponding Murphy basis element (cf. [44]) of the symmetric group algebra $K \mathfrak{S}_{\{2 f+1, \cdots, n\}}$. Then the set

$$
\left\{\begin{array}{c|c}
d_{1}{ }^{*} e_{1} e_{3} \cdots e_{2 f-1} m_{\mathfrak{s t}} d_{2} & 0 \leq f \leq[n / 2], \lambda \vdash n-2 f, \mathfrak{s}, \mathbf{t} \in \operatorname{Std}(\lambda), \\
d_{1}, d_{2} \in \mathfrak{D}_{f}
\end{array}\right\}
$$

is a cellular basis of the Brauer algebra $\mathfrak{B}_{n}(m)_{\mathbb{Z}}$.

As a consequence, by combining Lemma 4.1 and [21, (3.3)], we have

Corollary 4.2. With the above notations, the set

$$
\left\{\begin{array}{c|c}
d_{1}{ }^{*} e_{1} e_{3} \cdots e_{2 f-1} \sigma d_{2} & \begin{array}{c}
0 \leq f \leq[n / 2], \sigma \in \mathfrak{S}_{\{2 f+1, \cdots, n\}} \\
d_{1}, d_{2} \in \mathfrak{D}_{f}
\end{array}
\end{array}\right\}
$$

is a basis of the Brauer algebra $\mathfrak{B}_{n}(m)_{\mathbb{Z}}$.

From now on and until the end of this section, we shall regard the tensor space $V^{\otimes n}$ as a module over $\mathfrak{B}_{n}(m)$ via $\widehat{\varphi}$ (instead of $\varphi$ ). To prove the injectivity of $\widehat{\varphi}$, it suffices to show that the annihilator $\operatorname{ann}_{\mathfrak{B}_{n}(m)}\left(V^{\otimes n}\right)$ is $(0)$. Note that

$$
\operatorname{ann}_{\mathfrak{B}_{n}(m)}\left(V^{\otimes n}\right)=\bigcap_{v \in V^{\otimes n}} \operatorname{ann}_{\mathfrak{B}_{n}(m)}(v) .
$$

Thus it is enough to calculate $\operatorname{ann}_{\mathfrak{B}_{n}(m)}(v)$ for some set of chosen vectors $v \in V^{\otimes n}$ such that the intersection of annihilators is (0). We write

$$
\operatorname{ann}(v)=\operatorname{ann}_{\mathfrak{B}_{n}(m)}(v):=\left\{x \in \mathfrak{B}_{n}(m) \mid v x=0\right\} .
$$

For each integer $f$ with $0 \leq f \leq[n / 2]$, we denote by $B^{(f)}$ the two-sided ideal of $\mathfrak{B}_{n}(m)_{\mathbb{Z}}$ generated by $e_{1} e_{3} \cdots e_{2 f-1}$. Note that $B^{(f)}$ is spanned by all the Brauer diagrams which contain at least $2 f$ horizontal edges ( $f$ edges in each of the top and the bottom rows in the diagrams).

For $\underline{i} \in I(m, n)$, an ordered pair $(s, t)(1 \leq s<t \leq n)$ is called an orthogonal pair in $\underline{i}$ if $i_{s}=i_{t}$. Two ordered pairs $(s, t)$ and $(u, v)$ are called disjoint if $\{s, t\} \cap\{u, v\}=\emptyset$. We define the orthogonal length $\ell_{o}\left(v_{\underline{i}}\right)=\ell_{o}(\underline{i})$ to be the maximal number of disjoint orthogonal pairs $(s, t)$ in $\underline{i}$. Note that if $f>\ell_{o}\left(v_{\underline{i}}\right)$, then clearly $B^{(f)} \subseteq \operatorname{ann}\left(v_{\underline{i}}\right)$.

Lemma 4.3. $\quad \operatorname{ann}_{\mathfrak{B}_{n}(m)}\left(V^{\otimes n}\right) \subseteq B^{(1)}$.

Proof. Let $x \in \operatorname{ann}_{\mathfrak{B}_{n}(m)}\left(V^{\otimes n}\right)$. Then we can write $x=y+z$ where $y \in K \mathfrak{S}_{n}, z \in B^{(1)}$, because the set of diagrams with at least one horizontal edge spans $B^{(1)}$ (or else see Corollary 4.2).

Since $m \geq n$, the tensor $v:=v_{1} \otimes v_{2} \otimes \cdots \otimes v_{n}$ is well-defined and $\ell_{o}(v)=0$. It follows that $B^{(1)} \subseteq \operatorname{ann}(v)$. In particular, $v z=0$. Therefore $v x=0$ implies that $v y=0$. 
On the other hand, since $v_{1}, \cdots, v_{n}$ are pairwise distinct and $y \in K \mathfrak{S}_{n}$, it is clear that $v y=0$ implies that $y=0$. Therefore, we conclude that $x=z \in B^{(1)}$, as required.

Suppose that we have already shown $\operatorname{ann}_{\mathfrak{B}_{n}(m)}\left(V^{\otimes n}\right) \subseteq B^{(f)}$ for some natural number $1 \leq$ $f \leq[n / 2]$. We want to show that $\operatorname{ann}_{\mathfrak{B}_{n}(m)}\left(V^{\otimes n}\right) \subseteq B^{(f+1)}$. Let

$$
\underline{c}:=(1,1,2,2, \cdots, f, f) \text {. }
$$

We define

$$
I_{f}:=\left\{\underline{b}=\left(b_{1}, \cdots, b_{n-2 f}\right) \mid 2 f+1 \leq b_{1}<\cdots<b_{n-2 f} \leq m\right\} .
$$

It is clear that $\ell_{o}\left(v_{\underline{c}} \otimes v_{\underline{b}}\right)=f$ for all $\underline{b} \in I_{f}$.

Following [10, we consider the subgroup $\Pi$ of $\mathfrak{S}_{\{1, \cdots, 2 f\}} \leq \mathfrak{S}_{n}$ permuting the rows of $\mathfrak{t}^{\nu^{(1)}}$ but keeping the entries in the rows fixed. $\Pi$ normalizes the stabilizer $\mathfrak{S}_{\left(2^{f}\right)}$ of $\mathfrak{t}^{\nu^{(1)}}$ in $\mathfrak{S}_{2 f}$. We set $\Psi:=\mathfrak{S}_{\left(2^{f}\right)} \rtimes \Pi$. By [10, Lemma 3.7], we have

$$
\mathfrak{S}_{2 f}=\bigsqcup_{d \in \mathcal{D}_{f}} \Psi d
$$

where $\mathcal{D}_{f}:=\mathfrak{D}_{f} \bigcap \mathfrak{S}_{2 f}$, and "ப" means a disjoint union. Let $\mathcal{P}_{f}:=\left\{\left(i_{1}, \cdots, i_{2 f}\right) \mid 1 \leq i_{1}<\right.$ $\left.\cdots<i_{2 f} \leq n\right\}$. For each $J \in \mathcal{P}_{f}$, we use $d_{J}$ to denote the unique element in $\mathfrak{D}_{f}$ such that the first component of $\mathfrak{t}^{\nu} d_{J}$ is the tableau obtained by inserting the integers in $J$ in increasing order along successive rows in $\mathfrak{t}^{\nu^{(1)}}$. Let $\widetilde{\mathcal{D}}_{(2 f, n-2 f)}$ be the set of distinguished right coset representatives of $\mathfrak{S}_{(2 f, n-2 f)}$ in $\mathfrak{S}_{n}$. Clearly $d_{J} \in \widetilde{\mathcal{D}}_{(2 f, n-2 f)}$, and every element of $\widetilde{\mathcal{D}}_{(2 f, n-2 f)}$ is of the form $d_{J}$ for some $J \in \mathcal{P}_{f}$. By [10, Lemma 3.8], $\mathfrak{D}_{f}=\bigsqcup_{J \in \mathcal{P}_{f}} \mathcal{D}_{f} d_{J}$.

The proof of the next two lemmas is similar to [10, Lemma 3.9, Lemma 3.10] except some minor changes. For the reader's convenience, we include the proof here.

LEMmA 4.4. Let $\underline{b} \in I_{f}, v=v_{\underline{c}} \otimes v_{\underline{b}} \in V^{\otimes n}$. Let $1 \neq d \in \mathfrak{S}_{n}$. If either $d \notin \mathfrak{S}_{(2 f, n-2 f)}$ or $d \in \mathcal{D}_{f}$, then $d^{-1} z e_{1} e_{3} \cdots e_{2 f-1} \in \overline{\operatorname{ann}}(\bar{v})$ for any $z \in \Psi$.

Proof. If $d \notin \mathfrak{S}_{(2 f, n-2 f)}$. Then $d^{-1}$ is not an element of $\mathfrak{S}_{(2 f, n-2 f)}$ too. In particular, there is some $j, 2 f+1 \leq j \leq n$, such that $1 \leq j d^{-1} \leq 2 f$, and hence the basis vector $v_{b_{j}}$ with $2 f+1 \leq$ $b_{j} \leq m$ appears at position $j d^{-1}$ in $v d^{-1}$. However, $v_{b_{j}}$ occurs only once as a factor in $v d^{-1}$ and hence for any $z \in \Psi, 0=v d^{-1} z e_{j d^{-1}-1}$ if $j d^{-1}$ is even, $0=v d^{-1} z e_{j d^{-1}}$ if $j d^{-1}$ is odd. As the $e_{i}$ 's in $e_{1} e_{3} \cdots e_{2 f-1}$ commute we have $v d^{-1} z e_{1} e_{3} \cdots e_{2 f-1}=0$ in this case. If $d \in \mathcal{D}_{f}=\mathfrak{D}_{f} \cap \mathfrak{S}_{2 f}$, then $d$ and hence $d^{-1}$ as well is not contained in the subgroup $\Psi$ of $\mathfrak{S}_{2 f}$ defined above. Therefore there exists $j \in\{1,3, \cdots, 2 f-1\}$ such that $j d^{-1},(j+1) d^{-1}$ are not in the same row of $\mathfrak{t}^{\left(2^{f}\right)} d^{-1}$. Now we see similarly as above that $z e_{1} e_{3} \cdots e_{2 f-1}$ annihilates $v d^{-1}$ for any $z \in \Psi$.

LEMMA 4.5. Let $S$ be the subset

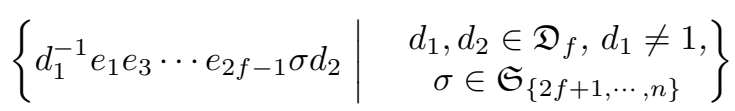

of the basis (4.2) of $\mathfrak{B}_{n}(m)$, and let $U$ be the subspace spanned by $S$. Then

$$
B^{(f)} \cap\left(\bigcap_{\underline{b} \in I_{f}} \operatorname{ann}\left(v_{\underline{c}} \otimes v_{\underline{b}}\right)\right)=B^{(f+1)} \oplus U .
$$


Proof. Since $\ell_{o}\left(v_{\underline{c}} \otimes v_{\underline{b}}\right)=f$, it follows that $B^{(f+1)} \subseteq \operatorname{ann}\left(v_{\underline{c}} \otimes v_{\underline{b}}\right)$. This, together with Lemma 4.4, shows that the right-hand side is contained in the left-hand side.

Now let $x \in B^{(f)} \cap\left(\cap_{\underline{b} \in I_{f}} \operatorname{ann}\left(v_{\underline{c}} \otimes v_{\underline{b}}\right)\right)$. Using Lemma 4.4 and the basis (4.2) of $\mathfrak{B}_{n}(-2 m)$, we may assume that $x=e_{1} e_{3} \cdots e_{2 f-1} \sum_{d \in \mathfrak{D}_{f}} z_{d} d$, where $\nu=\nu_{f}=\left(\left(2^{f}\right),(n-2 f)\right)$ and the coefficients $z_{d}$, where $d \in \mathfrak{D}_{f}$, are taken from $K \mathfrak{S}_{\{2 f+1, \cdots, n\}} \subseteq K \mathfrak{S}_{n}$. We then have to show $x=0$.

Fix $\underline{b} \in I_{f}$ and write $v=v_{\underline{c}} \otimes v_{\underline{b}}$. Let $\lambda^{(1)}, \lambda^{(2)}$ be the $G L_{m}$-weights of $v_{\underline{c}}$ and $v_{\underline{b}}$ respectively. Since $V^{\otimes n}$ is the direct sum of its $G L_{m}$-weight spaces, we conclude $(\bar{v} x)_{\mu}=0$ for all $\mu \in$ $\Lambda(m, n)$. In particular,

$$
\begin{aligned}
0 & =(v x)_{\lambda}=\left(\left(v_{\underline{c}} \otimes v_{\underline{b}}\right) x\right)_{\lambda}=\sum_{d \in \mathfrak{D}_{f}}\left(v_{\underline{c}} e_{1} e_{3} \cdots e_{2 f-1} \otimes v_{\underline{b}}\right)_{\lambda} z_{d} d \\
& =\sum_{d \in \mathfrak{D}_{f}}\left(\left(v_{\underline{c}} e_{1} e_{3} \cdots e_{2 f-1}\right)_{\lambda(1)} \otimes v_{\underline{b}}\right) z_{d} d .
\end{aligned}
$$

By definition, it is easy to see that

$$
\left(v_{\underline{c}} e_{1} e_{3} \cdots e_{2 f-1}\right)_{\lambda^{(1)}}=\sum_{y \in \Psi} v_{\underline{c}} y
$$

Let us denote this element by $\widehat{v}$. Then $\sum_{d \in \mathfrak{D}_{f}}\left(\widehat{v} \otimes v_{\underline{b}}\right) z_{d} d=0$.

We write $d=d_{1} d_{J}$, where $d_{1} \in \mathcal{D}_{f}, J \in \mathcal{P}_{f}$. Then

$$
\left(\widehat{v} \otimes v_{\underline{b}}\right) z_{d} d=\left(\widehat{v} \otimes v_{\underline{b}} z_{d}\right) d=\left(\widehat{v} \otimes v_{\underline{b}} z_{d}\right) d_{1} d_{J}=\left(\widehat{v} d_{1} \otimes v_{\underline{b}} z_{d_{1} d_{J}}\right) d_{J} .
$$

If $J, L \in \mathcal{P}_{f}, J \neq L$, choose $1 \leq l \leq n$ with $l \in J$ but $l \notin L$. Thus there exists an $j \in$ $\{1,2, \cdots, 2 f\}$ which is mapped by $d_{J}$ to $l$, but $(l) d_{L}^{-1}>2 f$. Note that for any $d \in \mathcal{D}_{f}$ all basis vectors $v_{i}$ occurring in $\widehat{v} d$ as factors have index in the set $\{1,2, \cdots, f\}$, and all those $v_{i}$ occurring in $v_{\underline{b}} z_{d d_{J}}$, respectively in $v_{\underline{b}} z_{d d_{L}}$, have index $i$ between $2 f+1$ and $\mathrm{m}$. Let $v_{i_{1}} \otimes \cdots \otimes v_{i_{n}}$ be a simple tensor involved in $\left(\widehat{v} d_{1} \otimes v_{\underline{b}} z_{d_{1} d_{J}}\right) d_{J}$ and $v_{j_{1}} \otimes \cdots \otimes v_{j_{n}}$ be a simple tensor involved in $\left(\widehat{v} d_{2} \otimes v_{b} z_{d_{2} d_{L}}\right) d_{L}$ for $d_{1}, d_{2} \in \bar{D}_{f}$. Then, by the above, we have that $2 f+1 \leq j_{l} \leq m$, and $v_{i_{l}}=v_{k}$ for some $1 \leq k \leq f$. Consequently the simple tensors $v_{\underline{i}}, \underline{i} \in I(m, n)$ involved in $\left\{\left(\widehat{v} d_{1} \otimes v_{\underline{b}} z_{d_{1} d_{J}}\right) d_{J}\right\}$ and in $\left\{\left(\widehat{v} d_{2} \otimes v_{\underline{b}} z_{d_{2} d_{L}}\right) d_{L}\right\}$ are disjoint, hence both sets are linearly independent. We conclude that $\sum_{d \in \mathcal{D}_{f}}\left(\widehat{v} d \otimes v_{\underline{b}} z_{d d_{J}}\right) d_{J}=0$ for each $J \in \mathcal{P}_{f}$, hence $\sum_{d_{1} \in \mathcal{D}_{f}} \widehat{v} d_{1} \otimes v_{\underline{b}} z_{d_{1} d_{J}}=0$.

Note that $\widehat{v} d_{1}$ is a linear combination of basis tensors $v_{\underline{i}}=v_{i_{1}} \otimes \cdots \otimes v_{i_{2 f}}$, with $\underline{i} \in \underline{c} \Psi d_{1}$, and that we obtain by varying $d_{1}$ through $\mathcal{D}_{f}$ precisely the partition of $\mathfrak{S}_{2 f}$ into $\Psi$-cosets. These are mutually disjoint. We conclude that the basic tensors involved in $\widehat{v} d_{1}$ are disjoint for different choices of $d_{1} \in \mathcal{D}_{f}$. Therefore, the equality $\sum_{d_{1} \in \mathcal{D}_{f}} \widehat{v} d_{1} \otimes v_{\underline{b}} z_{d_{1} d_{J}}=0$ implies that $\widehat{v} d_{1} \otimes v_{\underline{b}} z_{d_{1} d_{J}}=0$ for each fixed $d_{1} \in \mathcal{D}_{f}$. Now we vary $\underline{b} \in I_{f}$. The $K$-span of $\left\{v_{\underline{b}} \mid \underline{b} \in I_{f}\right\}$ is isomorphic to the tensor space $V^{\otimes n-2 f}$ for the symmetric group $\mathfrak{S}_{\{2 f+1, \cdots, n\}} \cong \mathfrak{S}_{n-2 f}$. Since $m-2 f \geq n-2 f$, hence $\mathfrak{S}_{\{2 f+1, \cdots, n\}}$ acts faithfully on it. This implies $z_{d_{1} d_{J}}=0$ for all $d_{1} \in \mathcal{D}_{f}, J \in \mathcal{P}_{f}$. Thus $x=0$ and the lemma is proved.

The following corollary can be proved in exactly the same way as in [10, Corollary 3.11].

Corollary 4.6. Let $d \in \mathfrak{D}_{f}, \nu=\nu_{f}$. Then

$$
B^{(f)} \cap\left(\bigcap_{\underline{b} \in I_{f}} \operatorname{ann}\left(\left(v_{\underline{c}} \otimes v_{\underline{b}}\right) d\right)\right)=B^{(f+1)} \oplus\left(\bigoplus K \tilde{d}_{1}^{-1} e_{1} e_{3} \cdots e_{2 f-1} \sigma d_{2}\right)
$$

where the rightmost direct sum is taken over all $\tilde{d}_{1}, d_{2} \in \mathfrak{D}_{f}$ such that $\tilde{d}_{1} \neq d$ and all $\sigma \in$ $\mathfrak{S}_{\{2 f+1, \cdots, n\}}$. Hence $B^{(f)} \cap\left(\bigcap_{d \in \mathfrak{D}_{f}} \bigcap_{\underline{b} \in I_{f}} \operatorname{ann}\left(\left(v_{\underline{c}} \otimes v_{\underline{b}}\right) d\right)\right)=B^{(f+1)}$. 
Proof of part b) in Theorem 1.2 in the case $m \geq n$ : We have seen that $\operatorname{ann}_{\mathfrak{B}_{n}(m)}\left(V^{\otimes n}\right) \subseteq B^{(1)}$, and the above Corollary implies that $\operatorname{ann}_{\mathfrak{B}_{n}(m)}\left(V^{\otimes n}\right) \subseteq B^{(f+1)}$ provided that $\operatorname{ann}_{\mathfrak{B}_{n}(m)}\left(V^{\otimes n}\right) \subseteq B^{(f)}$. Thus by induction on $f$ we have $\operatorname{ann}_{\mathfrak{B}_{n}(m)}\left(V^{\otimes n}\right) \subseteq B^{(f)}$ for all natural numbers $f$. Since $B^{(f+1)}=0$ for $f>[n / 2]$ it follows that $\operatorname{ann}_{\mathfrak{B}_{n}(m)}\left(V^{\otimes n}\right)=0$. In other words, $\widehat{\varphi}$ and hence $\varphi$ is injective if $m \geq n$. By comparing dimension, we deduce that $\varphi$ is an isomorphism onto

$$
\operatorname{End}_{K G O(V)}\left(V^{\otimes n}\right)=\operatorname{End}_{K O(V)}\left(V^{\otimes n}\right) .
$$

This completes the proof of part b) in Theorem 1.2 in the case $m \geq n$.

\section{Proof of part b) in Theorem 1.2 in the case $m<n$}

The purpose of this section is to give the proof the part b) in Theorem 1.2 in the case where $m<n$. Our approach is the same as that used in [10, Section 4].

To prove $\varphi\left(\mathfrak{B}_{n}(m)\right)=\operatorname{End}_{K O(V)}\left(V^{\otimes n}\right)$, we can assume without loss of generality that $K=$ $\bar{K}$ is algebraically closed. This is because, on the one hand, the $K$-dimension of $\operatorname{End}_{K O(V)}\left(V^{\otimes n}\right)$ does not depend on the choice of the infinite field $K$; on the other hand, the $\bar{K}$-dimension of $\varphi\left(\mathfrak{B}_{n}(m)_{\bar{K}}\right)$ is the same as the $K$-dimension of $\varphi\left(\mathfrak{B}_{n}(m)_{K}\right)$. We fix $m_{0} \in \mathbb{N}$ such that $m_{0} \geq m$ and $m_{0}-m$ is even. We denote by $\mathfrak{s o}_{m_{0}}, \mathfrak{s o}_{m}$ the special orthogonal Lie algebras over $\mathbb{C}$. Let $\widetilde{\mathfrak{g}}:=\mathfrak{s o}_{m_{0}}, \mathfrak{g}:=\mathfrak{s o}_{m}$. Recall that $\mathcal{R}=\mathbb{Z}[1 / 2]$. Let $\mathrm{U}_{\mathbb{Q}}$ (resp., $\mathrm{U}_{\mathcal{R}}$ ) be the universal enveloping algebra of $\mathfrak{g}$ over $\mathbb{Q}$ (resp., Kostant's $\mathcal{R}$-form in $\mathrm{U}_{\mathbb{Q}}$ ). Let $q$ be an indeterminant over $\mathcal{R}$. Let $\mathbf{U}_{\mathbb{Q}(q)}$ (resp., $\mathbf{U}_{\mathcal{R}}$ ) be the Drinfel'd-Jimbo quantized enveloping algebra of $\mathfrak{g}$ over $\mathbb{Q}(q)$ (resp., Lusztig's $\mathcal{R}\left[q, q^{-1}\right]$-form in $\left.\mathbf{U}_{\mathbb{Q}(q)}\right)$. Let $\mathrm{U}_{\mathrm{K}}:=\mathrm{U}_{\mathcal{R}} \otimes_{\mathcal{R}} \mathrm{K}, \mathbf{U}_{\mathrm{K}}:=\mathbf{U}_{\mathcal{R}} \otimes_{\mathcal{R}} \mathrm{K}$. By putting a " " on the head, we can define similar notations for $\widetilde{\mathfrak{g}}$.

Let $\widetilde{V}_{\mathcal{R}}$ be a free module of rank $m_{0}$ over $\mathcal{R}$. Assume that $\widetilde{V}_{\mathcal{R}}$ is equipped with a symmetric bilinear form $($,$) as well as an ordered basis \left\{v_{1}, v_{2}, \cdots, v_{m_{0}}\right\}$ satisfying $\left(v_{i}, v_{j}\right)=\delta_{i, m_{0}+1-j}$. For any commutative $\mathcal{R}$ algebra $K$, we set $\widetilde{V}_{K}:=\widetilde{V}_{\mathcal{R}} \otimes_{\mathcal{R}} K$. Let $\iota$ be the $K$-linear injection from $V_{K} \cong V_{\mathcal{R}} \otimes_{\mathcal{R}} K$ into $\widetilde{V}_{K}$ defined by

$$
\sum_{i=1}^{m} k_{i} v_{i} \mapsto \sum_{i=1}^{m} k_{i} v_{i+\left(m_{0}-m\right) / 2}, \quad \forall k_{1}, \cdots, k_{m} \in K .
$$

Let $\pi$ be the $K$-linear surjection from $\widetilde{V}_{K}$ onto $V_{K}$ defined by

$$
\sum_{i=1}^{m_{0}} k_{i} v_{i} \mapsto \sum_{i=1}^{m} k_{i+\left(m_{0}-m\right) / 2} v_{i}, \quad \forall k_{1}, \cdots, k_{m_{0}} \in K
$$

Then, $\iota$ induces an identification of $\mathfrak{g}$ as a subalgebra of $\widetilde{\mathfrak{g}}$, and also an identification of $S O_{m}(K)$ (resp., $O_{m}(K)$ ) as a subgroup of $S O_{m_{0}}(K)$ (resp., $O_{m_{0}}(K)$ ). Henceforth, we fix these embeddings. The following result is well-known (cf. [33. Part I, Lemma 7.16, 7.17(6)]).

LEMMA 5.1. We have

$$
\begin{aligned}
& \operatorname{End}_{\mathrm{U}_{\mathrm{K}}(\widetilde{\mathfrak{g}})}\left(\widetilde{V}_{K}^{\otimes n}\right)=\operatorname{End}_{K S O_{m_{0}}(K)}\left(\widetilde{V}_{K}^{\otimes n}\right), \\
& \operatorname{End}_{\mathrm{U}_{\mathrm{K}}(\mathfrak{g})}\left(V_{K}^{\otimes n}\right)=\operatorname{End}_{K S O_{m}(K)}\left(V_{K}^{\otimes n}\right) .
\end{aligned}
$$

Note that the homomorphism $\iota$ and $\pi$ naturally induce a linear map

$$
\begin{aligned}
\Theta_{0}: \operatorname{End}_{K S O_{m_{0}}(K)}\left(\widetilde{V}_{K}^{\otimes n}\right) & \rightarrow \operatorname{End}_{K S O_{m}(K)}\left(V_{K}^{\otimes n}\right) \\
f & \mapsto \pi \circ f \circ \iota .
\end{aligned}
$$


By restriction, we get a linear map (again denoted by $\left.\Theta_{0}\right)$ from $\operatorname{End}_{K O_{m_{0}}(K)}\left(\widetilde{V}_{K}^{\otimes n}\right)$ to $\operatorname{End}_{K O_{m}(K)}\left(V_{K}^{\otimes n}\right)$. Note that, $\Theta_{0}$ is in general not an algebra map.

\section{LEMma 5.2. We have}

(1) the map

$$
\Theta_{0}: \operatorname{End}_{K S O_{m_{0}}(K)}\left(\widetilde{V}_{K}^{\otimes n}\right) \rightarrow \operatorname{End}_{K S O_{m}(K)}\left(V_{K}^{\otimes n}\right)
$$

is surjective.

(2) the map

$$
\Theta_{0}: \operatorname{End}_{K O_{m_{0}}(K)}\left(\widetilde{V}_{K}^{\otimes n}\right) \rightarrow \operatorname{End}_{K O_{m}(K)}\left(V_{K}^{\otimes n}\right)
$$

is surjective.

Proof. We first prove (1). By Lemma 5.1, it suffices to show that the map

$$
\Theta_{0}: \operatorname{End}_{\mathrm{U}_{\mathrm{K}}(\widetilde{\mathfrak{g}})}\left(\widetilde{V}_{K}^{\otimes n}\right) \rightarrow \operatorname{End}_{\mathrm{U}_{\mathrm{K}}(\mathfrak{g})}\left(V_{K}^{\otimes n}\right)
$$

is surjective. The same argument used in [10, Section 4] still works (except that we use a slightly different embedding $\mathfrak{g} \hookrightarrow \widetilde{\mathfrak{g}}$ here). So we shall give only a sketch here. Recall that for a module $M$ over a Hopf algebra $H, M^{H}:=\left\{x \in M \mid h x=\varepsilon_{H} x, \forall h \in H\right\}$. We have the following commutative diagram

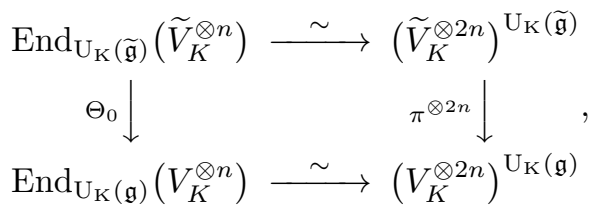

where the two horizontal maps are natural isomorphisms. Therefore, it suffices to show that

$$
\pi^{\otimes 2 n}\left(\left(\widetilde{V}_{K}^{\otimes 2 n}\right)^{\mathrm{U}_{\mathrm{K}}(\widetilde{\mathfrak{g}})}\right)=\left(V_{K}^{\otimes 2 n}\right)^{\mathrm{U}_{\mathrm{K}}(\mathfrak{g})} .
$$

Since (by the theory of tilting modules) all the maps and modules are defined over $\mathcal{R}$, it suffices to prove the above equality with $K$ replaced by $\mathcal{R}$. Let $\widetilde{M}[\neq 0]_{\mathcal{R}}, M[\neq 0]_{\mathcal{R}}, B[0], \widetilde{B}[0]$ be the notations for $\mathfrak{g}, \widetilde{\mathfrak{g}}$ which is defined in a similar way as in [10, Section 4]. We have the following commutative diagram.

$$
\begin{aligned}
\left(\widetilde{V}_{\mathcal{R}}^{\otimes 2 n}\right)^{\mathrm{U}_{\mathcal{R}}(\widetilde{\mathfrak{g}})} \stackrel{\sim}{\longrightarrow}\left(\widetilde{V}_{\mathcal{R}}^{\otimes 2 n} / \widetilde{M}[\neq 0]_{\mathcal{R}}\right)^{*} \longrightarrow\left(\widetilde{V}_{\mathcal{R}}^{\otimes 2 n}\right)^{*} \\
\pi^{\otimes 2 n} \downarrow \\
\left(V_{\mathcal{R}}^{\otimes 2 n}\right)^{\mathrm{U}_{\mathcal{R}}(\mathfrak{g})} \longrightarrow\left(\iota^{\otimes 2 n}\right)^{*} \downarrow \\
\longrightarrow\left(V_{\mathcal{R}}^{\otimes 2 n} / M[\neq 0]_{\mathcal{R}}\right)^{*} \longrightarrow\left(V_{\mathcal{R}}^{\otimes 2 n}\right)^{*}
\end{aligned}
$$

Hence it suffices to show that the rightmost vertical map is surjective.

Let

$$
\begin{aligned}
& J_{0}:=\left\{\left(i_{1}, \cdots, i_{2 n}\right) \in I(m, 2 n) \mid w_{i_{1}} \diamond \cdots \diamond w_{i_{2 n}} \in B[0]\right\}, \\
& \widetilde{J}_{0}:=\left\{\left(i_{1}, \cdots, i_{2 n}\right) \in I\left(m_{0}, 2 n\right) \mid \tilde{w}_{i_{1}} \widetilde{\diamond} \cdots \tilde{\diamond} \tilde{w}_{i_{2 n}} \in \widetilde{B}[0]\right\},
\end{aligned}
$$

where $\left\{w_{1}, \cdots, w_{m}\right\}$ (resp., $\left\{\tilde{w}_{1}, \cdots, \tilde{w}_{m}\right\}$ ) are canonical bases of $V_{\mathcal{R}}$ (resp., of $\widetilde{V}_{\mathcal{R}}$ ), " $\diamond$ " is the notation in [35, (27.3.2)], and " $\widetilde{\diamond}$ " is the similar notation for $\widetilde{\mathfrak{g}}$. As in [10, Corollary 4.5], it is clear that the set

$$
\left\{w_{i_{1}} \otimes \cdots \otimes w_{i_{2 n}}+M[\neq 0]_{\mathcal{R}} \mid\left(i_{1}, \cdots, i_{2 n}\right) \in J_{0}\right\}
$$


forms an $\mathcal{R}$-basis of $V_{\mathcal{R}}^{\otimes 2 n} / M[\neq 0]_{\mathcal{R}}$, and the set

$$
\left\{\tilde{w}_{i_{1}} \otimes \cdots \otimes \tilde{w}_{i_{2 n}}+\widetilde{M}[\neq 0]_{\mathcal{R}} \mid\left(i_{1}, \cdots, i_{2 n}\right) \in \widetilde{J}_{0}\right\}
$$

forms an $\mathcal{R}$-basis of $\widetilde{V}_{\mathcal{R}}^{\otimes 2 n} / \widetilde{M}[\neq 0]_{\mathcal{R}}$. We set

$$
J_{0}\left[\left(m_{0}-m\right) / 2\right]:=\left\{\left(\left(m_{0}-m\right) / 2+i_{1}, \cdots,\left(m_{0}-m\right) / 2+i_{2 n}\right) \mid\left(i_{1}, \cdots, i_{2 n}\right) \in J_{0}\right\} .
$$

Using the same argument as in the proof of [10. Theorem 4.7], we can show that $J_{0}\left[\left(m_{0}-m\right) / 2\right] \subseteq \widetilde{J}_{0}$. This implies that $\iota^{\otimes 2 n} \operatorname{maps} V_{\mathcal{R}}^{\otimes 2 n} / M[\neq 0]_{\mathcal{R}}$ isomorphically onto an $\mathcal{R}$-direct summand of $\widetilde{V}_{\mathcal{R}}^{\otimes 2 n} / \widetilde{M}[\neq 0]_{\mathcal{R}}$. It follows that

$$
\left(\iota^{\otimes 2 n}\right)^{*}\left(\left(\widetilde{V}_{\mathcal{R}}^{\otimes 2 n} / \widetilde{M}[\neq 0]_{\mathcal{R}}\right)^{*}\right)=\left(V_{\mathcal{R}}^{\otimes 2 n} / M[\neq 0]_{\mathcal{R}}\right)^{*},
$$

as required. This proves (1).

For (2), we note that if $m$ is odd, then $m_{0}$ is odd too (as $m_{0}-m$ is even). In this case, since $\theta$ acts as a scalar $(1$ or -1$)$ on $V_{K}^{\otimes n}$, it is readily seen that

$$
\begin{aligned}
\operatorname{End}_{K O_{m_{0}}(K)}\left(\widetilde{V}_{K}^{\otimes n}\right) & =\operatorname{End}_{K S O_{m_{0}}(K)}\left(\widetilde{V}_{K}^{\otimes n}\right), \\
\operatorname{End}_{K O_{m}(K)}\left(V_{K}^{\otimes n}\right) & =\operatorname{End}_{K S O_{m}(K)}\left(V_{K}^{\otimes n}\right) .
\end{aligned}
$$

Therefore, by (1), we know $\Theta_{0}$ is surjective in this case. Now we assume that $m$ is even, then $m_{0}$ is also even. We have the following commutative diagram.

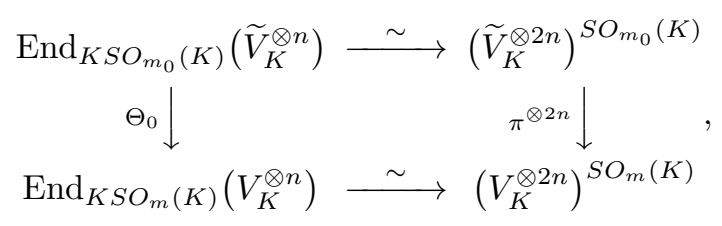

which implies (by (1)) that

$$
\pi^{\otimes 2 n}\left(\left(\widetilde{V}_{K}^{\otimes 2 n}\right)^{S O_{m_{0}}(K)}\right)=\left(V_{K}^{\otimes 2 n}\right)^{S O_{m}(K)} .
$$

Since $\theta$ normalizes $S O_{m}(K), \theta$ must stabilize $\left(V_{K}^{\otimes 2 n}\right)^{S O_{m}(K)}$. As $\theta^{2}=1$ and $1 \neq-1$, it follows that $\theta$ acts semisimply on $\left(V_{K}^{\otimes 2 n}\right)^{S O_{m}(K)}$ with two eigenvalues $1,-1$, and $\left(V_{K}^{\otimes 2 n}\right)^{O_{m}(K)}$ is nothing but its eigenspace belonging to 1 . By (5.3),$\pi^{\otimes 2 n}$ must map the eigenspace of $\left(\widetilde{V}_{K}^{\otimes 2 n}\right)^{S O_{m_{0}}(K)}$ belonging to 1 surjectively onto the eigenspace of $\left(V_{K}^{\otimes 2 n}\right)^{S O_{m}(K)}$ belonging to 1 . In other words,

$$
\pi^{\otimes 2 n}\left(\left(\widetilde{V}_{K}^{\otimes 2 n}\right)^{O_{m_{0}}(K)}\right)=\left(V_{K}^{\otimes 2 n}\right)^{O_{m}(K)}
$$

Now the surjectivity of $\Theta_{0}$ in this case follows directly from the following commutative diagram.

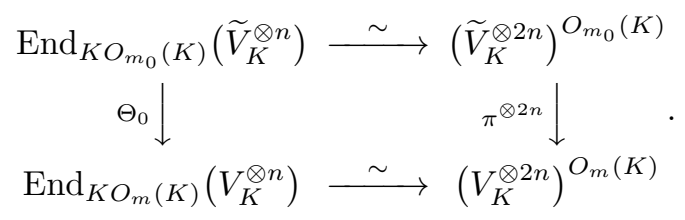

This completes the proof of the lemma.

${ }^{\dagger}$ Note that we get a shift here because we have used a slight different embedding $\mathfrak{g} \hookrightarrow \widetilde{\mathfrak{g}}$. 
Recall that the orthogonal bilinear form on $V$ determines an $O_{m}(K)$-isomorphism $V_{K} \cong V_{K}^{*}$. Therefore, there is an isomorphism

$$
\operatorname{End}_{K}\left(V_{K}^{\otimes n}\right) \cong V_{K}^{\otimes n} \otimes\left(V_{K}^{\otimes n}\right)^{*} \cong\left(V_{K}^{\otimes 2 n}\right)^{*}
$$

such that for any given $\underline{i}=\left(i_{1}, \cdots, i_{n}\right), \underline{j}=\left(j_{1}, \cdots, j_{n}\right) \in I(m, n)$, the map which sends $v_{\underline{l}}:=$ $v_{l_{1}} \otimes \cdots \otimes v_{l_{n}}$ to $\delta_{\underline{i}, \underline{\underline{l}} v_{\underline{j}}}$ corresponds to the linear function

$$
\begin{array}{r}
v_{k_{1}} \otimes v_{k_{2}} \otimes \cdots \otimes v_{k_{2 n}} \mapsto \delta_{m+1-j_{1}, k_{2 n}} \delta_{m+1-j_{2}, k_{2 n-1}} \cdots \delta_{m+1-j_{n}, k_{n+1}} \\
\times \delta_{k_{1}, i_{1}} \delta_{k_{2}, i_{2}} \cdots \delta_{k_{n}, i_{n}},
\end{array}
$$

for any $\left(k_{1}, \cdots, k_{2 n}\right) \in I(m, 2 n)$. The symmetric group $\mathfrak{S}_{2 n}$ acts on $V_{K}^{\otimes 2 n}$ by place permutation, hence also acts on $\left(V_{K}^{\otimes 2 n}\right)^{*} \cong \operatorname{End}_{K}\left(V_{K}^{\otimes n}\right)$. Similar results also hold for $\widetilde{V}_{K}$ (with $m$ replaced by $\left.m_{0}\right)$.

Let $D \in \mathrm{Bd}_{n}$. We can write $D=D_{\underline{i}, \underline{j}}$, where

$$
\underline{i}=\left(i_{1}, \cdots, i_{n}\right), \underline{j}=\left(j_{1}, \cdots, j_{n}\right),
$$

such that $\left(i_{1}, j_{1}, i_{2}, j_{2}, \cdots, i_{n}, j_{n}\right)$ is a permutation of $(1,2,3, \cdots, 2 n)$, and for each integer $1 \leq s \leq n$, the vertex labelled by $i_{s}$ is connected with the vertex labelled by $j_{s}$.

Lemma 5.5. (cf. 17, 23, Proposition 1.6], 34]) With the notations as above and (5.4) in mind, for any $w_{1}, \cdots, w_{2 n} \in V_{K}$, we have that

$$
\varphi\left(D_{\underline{i}, \underline{j}}\right)\left(w_{1} \otimes \cdots \otimes w_{2 n}\right)=\prod_{s=1}^{n}\left(w_{i_{s}}, w_{j_{s}}\right) .
$$

Furthermore, $\varphi$ is a $\mathfrak{S}_{2 n}$-module homomorphism. Similar results also hold for $\widetilde{V}_{K}$.

Proof. Let $d_{1}^{-1} e_{1} e_{3} \cdots e_{2 f-1} \sigma d_{2}$ be the basis element which corresponds to the Brauer diagram $D$, where $f$ be an integer with $0 \leq f \leq[n / 2], \sigma \in \mathfrak{S}_{\{2 f+1, \cdots, n\}}$ and $d_{1}, d_{2} \in \mathfrak{D}_{f}$, $\nu_{f}:=\left(\left(2^{f}\right),(n-2 f)\right) \vdash n$. Then the top horizontal edges of $D$ connect $(2 i-1) d_{1}$ and $(2 i) d_{1}$, the bottom horizontal edges of $D$ connect $(2 i-1) d_{2}$ and $(2 i) d_{2}$, for $i=1,2, \cdots, f$, and the vertical edges of $D$ connect $(j) d_{1}$ and $(j) \sigma d_{2}$, for $j=2 f+1,2 f+2, \cdots, n$.

Let $\underline{i} \in I(m, n)$. By our definitions of $\varphi$ and the set $\mathfrak{D}_{f}$, the action of

$$
D=d_{1}^{-1} e_{1} e_{3} \cdots e_{2 f-1} \sigma d_{2}
$$

on $v_{\underline{i}}$ can be described as follows. Let $\left(a_{1}, b_{1}\right), \cdots,\left(a_{f}, b_{f}\right)$ be the set of all the horizontal edges in the top row of $D$, where $a_{s}<b_{s}$ for each $s$ and $a_{1}<a_{2}<\cdots<a_{f}$. Let $\left(c_{1}, d_{1}\right), \cdots,\left(c_{f}, d_{f}\right)$ be the set of all the horizontal edges in the bottom row of $D$, where $c_{s}<d_{s}$ for each $s$ and $c_{1}<c_{2}<\cdots<c_{f}$. Then for each integer $s$ with $1 \leq s \leq f$, the $\left(c_{s}, d_{s}\right)$ th position of $v_{\underline{i}} D$ is the following sum:

$$
\delta_{i_{a_{s}}, m+1-i_{b_{s}}} \sum_{k=1}^{m}\left(v_{k} \otimes v_{k^{\prime}}\right) .
$$

We list those vertices in the top row of $D$ which are not connected with horizontal edges from left to right as $i_{k_{2 f+1}}, i_{k_{2 f+2}}, \cdots, i_{k_{n}}$. Then, for each integer $s$ with $2 f+1 \leq s \leq n$, the $\left(s \sigma d_{2}\right)$ th position of $v_{\underline{i}} D$ is $v_{i_{k_{s}}}$. Now it is easy to verify directly that $\varphi\left(D_{\underline{i}, j}\right)\left(w_{1} \otimes \cdots \otimes w_{2 n}\right)=$ $\prod_{s=1}^{n}\left(w_{i_{s}}, w_{j_{s}}\right)$, from which we see immediately that $\varphi$ is a $\mathfrak{S}_{2 n}$-module homomorphism.

We define a linear isomorphism $\Theta_{1}$ from the $\mathfrak{B}_{n}\left(m_{0}\right)$ onto $\mathfrak{B}_{n}(m)$ as follows:

$$
\Theta_{1}\left(\widetilde{d}_{1}^{-1} \widetilde{e}_{1} \widetilde{e}_{3} \cdots \widetilde{e}_{2 f-1} \widetilde{\sigma} \widetilde{d}_{2}\right)=d_{1}{ }^{-1} e_{1} e_{3} \cdots e_{2 f-1} \sigma d_{2},
$$


for each $0 \leq f \leq[n / 2], \lambda \vdash n-2 f, d_{1}, d_{2} \in \mathfrak{D}_{f}$.

Lemma 5.6. The following diagram of maps

is commutative.

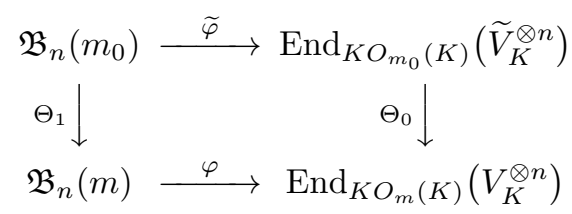

Proof. This follows directly from Lemma 5.5 .

Proof of Part b) in Theorem 1.2 in the case $m<n$ : Since $m_{0} \geq n$, by the main result in last section, we know that $\widetilde{\varphi}$ is surjective. Since $\Theta_{1}$ is a linear isomorphism, and by Lemma $5.2 \Theta_{0}$ is also surjective, the commutativity of the diagram in Lemma 5.6 immediately implies that $\varphi$ is also surjective. This completes the proof of Part b) in Theorem 1.2 in the case $m<n$.

\section{The $\mathfrak{S}_{2 n}$-action on $\mathfrak{B}_{n}(x)$}

In this section, we shall first introduce (cf. [22]) the right sign permutation action of the symmetric group $\mathfrak{S}_{2 n}$ on the set $\mathrm{Bd}_{n}$. Then we shall construct a new $\mathbb{Z}$-basis for the resulting right $\mathfrak{S}_{2 n}$-module, which yields filtrations of $\mathfrak{B}_{n}(x)$ by right $\mathfrak{S}_{2 n}$-modules. Certain submodules occurring in this filtration will play a central role in the next section.

For any fixed-point-free involution $\sigma$ in the symmetric group $\mathfrak{S}_{2 n}$, the conjugate $w^{-1} \sigma w$ of $\sigma$ by $w \in \mathfrak{S}_{2 n}$ is still a fixed-point-free involution. Therefore, we have a right action of the symmetric group $\mathfrak{S}_{2 n}$ on the set of fixed-point-free involutions in $\mathfrak{S}_{2 n}$. Note that the set $\mathrm{Bd}_{n}$ of Brauer $n$-diagrams can be naturally identified with the set of fixed-point-free involutions in $\mathfrak{S}_{2 n}$. Hence we get (cf. [22, [30]) a right permutation action of the symmetric group $\mathfrak{S}_{2 n}$ on the set $\mathrm{Bd}_{n}$ of Brauer $n$-diagrams. We use "* " to denote this right permutation action. Let $\mathfrak{B}_{n}$ denote the free $\mathbb{Z}$-module spanned by all the Brauer $n$-diagrams in $\operatorname{Bd}_{n}$. The right sign permutation action of $\mathfrak{S}_{2 n}$ on $\mathfrak{B}_{n}$ is defined by

$$
D \star w:=(-1)^{\ell(w)} D * w .
$$

We shall adopt the following labelling of the vertices in each Brauer diagram. Namely, for each Brauer $n$-diagram $D$, we shall label the vertices in the top row of $D$ by integers $1,2,3, \cdots, n$ from left to right, and label the vertices in the bottom row of $D$ by integers $n+1, n+2, n+$ $3, \cdots, 2 n$ from right to left (see Figure 6.1 for an example for $n=5$ ). This way of labelling is more suitable for studying the sign permutation action from $\mathfrak{S}_{2 n}$.

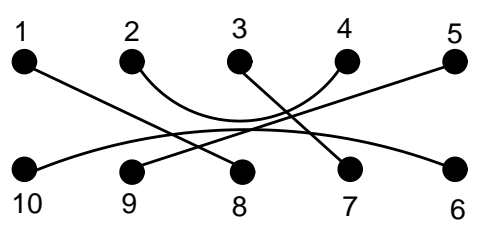

Figure 6.1 
For any commutative $\mathbb{Z}$-algebra $R$, we use $\mathfrak{B}_{n, R}$ to denote the free $R$-module spanned by all the Brauer $n$-diagrams in $\mathrm{Bd}_{n}$. Then $\mathfrak{B}_{n, R}$ becomes a right $R\left[\mathfrak{S}_{2 n}\right]$-module. Clearly, there is a canonical isomorphism $\mathfrak{B}_{n, R} \cong R \otimes_{\mathbb{Z}} \mathfrak{B}_{n}$, which is also a right $R\left[\mathfrak{S}_{2 n}\right]$-module isomorphism. Taking $R=\mathbb{Z}[x]$, we deduce that the Brauer algebra $\mathfrak{B}_{n}(x)$ becomes a right $\mathbb{Z}[x]\left[\mathfrak{S}_{2 n}\right]$-module.

For any $1 \leq i \leq 2 n$, we define $\gamma(i):=2 n+1-i$. Then $\gamma$ is an involution on $\{1,2, \cdots, 2 n\}$. It is well-known that the subgroup

$$
\left\{w \in \mathfrak{S}_{2 n} \mid(\gamma(a)) w=\gamma(a w) \text { for any } 1 \leq a \leq 2 n\right\}
$$

is isomorphic to the wreath product $\mathbb{Z}_{2} 2 \mathfrak{S}_{n}$ of $\mathbb{Z}_{2}$ and $\mathfrak{S}_{n}$, which is a Weyl group of type $B_{n}$ (cf. [29]).

For any commutative $\mathbb{Z}$-algebra $R$, let $1_{R}$ denote the rank one trivial representation of $R\left[\mathbb{Z}_{2}\right.$ ? $\left.\mathfrak{S}_{n}\right]$. By [30 Lemma 2.1], under the right permutation action $*$, there is a right $R\left[\mathfrak{S}_{2 n}\right]$-module isomorphism

$$
\mathfrak{B}_{n, R} \cong \operatorname{Ind}_{R\left[\mathbb{Z}_{2}\left(\mathfrak{S}_{n}\right]\right.}^{R\left[\mathfrak{G}_{2 n}\right]} 1_{R}
$$

Let $\mathrm{SGN}_{R}$ be the rank one sign representation of $\mathfrak{S}_{2 n}$.

Lemma 6.1. Let $\mathfrak{S}_{2 n}$ act on $\mathfrak{B}_{n, R}$ via the right sign permutation action " $\star$ " . Then there is a right $R\left[\mathfrak{S}_{2 n}\right]$-module isomorphism

$$
\mathfrak{B}_{n, R} \cong \mathrm{SGN}_{R} \otimes \operatorname{Ind}_{R\left[\mathbb{Z}_{2}\left(\mathfrak{S}_{n}\right]\right.}^{R\left[\mathfrak{G}_{2 n}\right]} 1_{R}
$$

Let $k$ be a positive integer. A sequence of nonnegative integers $\lambda=\left(\lambda_{1}, \lambda_{2}, \cdots\right)$ is said to be a composition of $k$ (denoted by $\lambda \vDash k$ ) if $\sum_{i \geq 1} \lambda_{i}=k$. A composition $\lambda=\left(\lambda_{1}, \lambda_{2}, \cdots\right)$ is said to be a partition of $k$ (denoted by $\lambda \vdash k$ ) if $\lambda_{1} \geq \lambda_{2} \geq \cdots$. The conjugate of $\lambda$ is defined to be a partition $\lambda^{\prime}=\left(\lambda_{1}^{\prime}, \lambda_{2}^{\prime}, \cdots\right)$, where $\lambda_{j}^{\prime}:=\#\left\{i \mid \lambda_{i} \geq j\right\}$ for $j=1,2, \cdots$. Denote by $\mathcal{P}_{n}$ the set of partitions of $n$. For any partition $\mu$ of $2 n$, denote by $S^{\mu}$ the associated Specht module over $\mathfrak{S}_{2 n}$. The notion of Specht modules we use here is the same as that introduced in [44. In particular, $S^{(2 n)}$ is the one-dimensional trivial representation of $\mathfrak{S}_{2 n}$, while $S^{\left(1^{2 n}\right)}$ is the one dimensional sign representation of $\mathfrak{S}_{2 n}$. For any commutative $\mathbb{Z}$-algebra $R$, we write $S_{R}^{\mu}:=R \otimes_{\mathbb{Z}} S^{\mu}$. Then $\left\{S_{\mathbb{Q}}^{\mu} \mid \mu \vdash 2 n\right\}$ is a complete set of pairwise non-isomorphic simple $\mathbb{Q}\left[\mathfrak{S}_{2 n}\right]$-modules.

For any composition $\lambda=\left(\lambda_{1}, \cdots, \lambda_{s}\right)$ of $n$, let $2 \lambda:=\left(2 \lambda_{1}, \cdots, 2 \lambda_{s}\right)$, which is a composition of $2 n$. We define $2 \mathcal{P}_{n}:=\left\{2 \lambda \mid \lambda \in \mathcal{P}_{n}\right\}$.

Lemma 6.2. Let $\mathfrak{S}_{2 n}$ act on $\mathfrak{B}_{n, \mathbb{Q}}$ via the right sign permutation action " $\star$ " . Then there is a right $\mathbb{Q}\left[\mathfrak{S}_{2 n}\right]$-module isomorphism

$$
\mathfrak{B}_{n, \mathbb{Q}} \cong \bigoplus_{\lambda \in 2 \mathcal{P}_{n}} S_{\mathbb{Q}}^{\lambda^{\prime}}
$$

Proof. This follows from [41, Chapter VII, (2.4)] (see [30, Lemma 2.2]).

For any non-negative integers $a, b$ with $a+b \leq n$, we denote by $\mathrm{Bd}_{(b)}^{(a)}$ the set of all the Brauer diagrams in $\mathrm{Bd}_{n}$ which satisfy the following two conditions:

1) for any integer $i$ with $i \leq a$ or $i>a+b$, the vertex labelled by $i$ is connected with the vertex labelled by $\gamma(i)$;

2) for any integer $i$ with $a<i \leq a+b$, the vertex labelled by $i$ is connected with the vertex labelled by $\gamma(j)$ for some integer $j$ with $a<j \leq a+b$. 
Note that one can naturally identify any Brauer diagram $D$ in $\operatorname{Bd}_{(b)}^{(a)}$ with an element $w(D)$ in $\mathfrak{S}_{(a+1, a+2, \cdots, a+b)}$. Thus we can attach a sign $\epsilon(D):=(-1)^{\ell(w(D))}$ to each Brauer diagram in $\operatorname{Bd}_{(b)}^{(a)}$, where $\ell(?)$ is the usual length function. We define

$$
Y_{(b)}^{(a)}:=\sum_{D \in \mathrm{Bd}_{(b)}^{(a)}} \epsilon(D) D
$$

For each integer $k$ with $0 \leq k \leq n$, we set $Y_{(k)}:=Y_{(k)}^{(0)}$.

Definition 6.3. Let $\lambda=\left(\lambda_{1}, \cdots, \lambda_{s}\right)$ be a partition of $n$. We define

$$
Y_{\lambda}:=Y_{\left(\lambda_{1}\right)}^{(0)} Y_{\left(\lambda_{2}\right)}^{\left(\lambda_{1}\right)} \cdots Y_{\left(\lambda_{s}\right)}^{\left(\lambda_{1}+\lambda_{2}+\cdots+\lambda_{s-1}\right)} \in \mathfrak{B}_{n}
$$

We identify $\mathfrak{S}_{n}$ with the set of Brauer $n$-diagrams in $\mathfrak{B}_{n}$ which contain no horizontal edges. Let $\mathfrak{S}_{\lambda}$ be its Young subgroup corresponding to $\lambda$. Then we actually have $Y_{\lambda} \in \mathfrak{S}_{\lambda} \subset \mathfrak{S}_{n} \subset \mathfrak{B}_{n}$. For any positive integer $k$ and any composition $\mu=\left(\mu_{1}, \cdots, \mu_{s}\right)$ of $k$, the Young diagram of $\mu$ is defined to be the set $[\mu]:=\left\{(a, b) \mid 1 \leq a \leq s, 1 \leq b \leq \mu_{a}\right\}$. The elements of $[\mu]$ are called nodes of $\mu$. A $\mu$-tableau $\mathfrak{t}$ is defined to be a bijective map from the Young diagram $[\mu]$ onto the set $\{1,2, \cdots, k\}$. For each integer $a$ with $1 \leq a \leq k$, we define $\operatorname{res}_{\mathfrak{t}}(a)=j-i$ if $\mathfrak{t}(i, j)=a$. We denote by $\mathfrak{t}^{\mu}$ the $\mu$-tableau in which the numbers $1,2, \cdots, k$ appear in order along successive rows. We denote by $\mathfrak{t}_{\mu}$ the $\mu$-tableau in which the numbers $1,2, \cdots, k$ appear in order along successive columns. The row stabilizer of $\mathfrak{t}^{\mu}$, denoted by $\mathfrak{S}_{\mu}$, is the Young subgroup of $\mathfrak{S}_{k}$ corresponding to $\mu$. We define

$$
x_{\mu}=\sum_{w \in \mathfrak{S}_{\mu}} w, \quad y_{\mu}=\sum_{w \in \mathfrak{S}_{\mu}}(-1)^{\ell(w)} w .
$$

Let $w_{\mu} \in \mathfrak{S}_{k}$ be such that $\mathfrak{t}^{\mu} w_{\mu}=\mathfrak{t}_{\mu}$. For example, if $k=8, \mu=(3,3,1,1)$, then

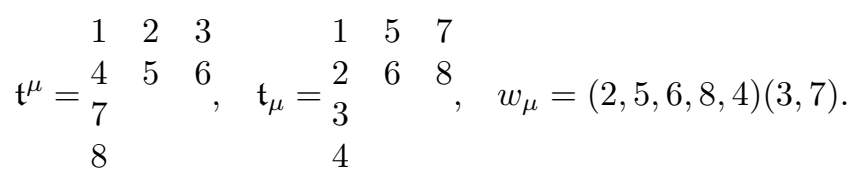

For any partition $\lambda=\left(\lambda_{1}, \lambda_{2}, \cdots, \lambda_{s}\right)$ of $n$, we define

$$
\widehat{\lambda}:=\left(\lambda_{1}, \lambda_{2}, \cdots, \lambda_{s}, \lambda_{s}, \cdots, \lambda_{2}, \lambda_{1}\right),
$$

which is a composition of $2 n$. Let $\mathfrak{S}_{\widehat{\lambda}}$ be the Young subgroup of $\mathfrak{S}_{2 n}$ corresponding to $\widehat{\lambda}$.

Lemma 6.4. Let $\lambda=\left(\lambda_{1}, \cdots, \lambda_{s}\right)$ be a partition of $n$. Then for any $w \in \mathfrak{S}_{\widehat{\lambda}}$, we have that

$$
Y_{\lambda} \star w=Y_{\lambda} .
$$

Proof. Since the elements $Y_{\left(\lambda_{1}\right)}^{(0)}, Y_{\left(\lambda_{2}\right)}^{\left(\lambda_{1}\right)}, \cdots, Y_{\left(\lambda_{s}\right)}^{\left(\lambda_{1}+\lambda_{2}+\cdots+\lambda_{s-1}\right)}$ pairwise commute with one another, the lemma follows directly from definition.

Let $k$ be a positive integer and $\mu$ be a composition of $k$. Recall that a $\mu$-tableau $\mathfrak{t}$ is called row standard if the numbers increase along rows. We use $\operatorname{RowStd}(\mu)$ to denote the set of all the row-standard $\mu$-tableaux. Suppose $\mu$ is a partition of $k$. Then $\mathfrak{t}$ is called column standard if the numbers increase down columns, and standard if it is both row and column standard. We use $\operatorname{Std}(\mu)$ to denote the set of all the standard $\mu$-tableaux. 
Note that every partition in the set $\left(2 \mathcal{P}_{n}\right)^{\prime}$ is of the form

$$
\widetilde{\nu}:=\left(\nu_{1}, \nu_{1}, \nu_{2}, \nu_{2}, \cdots, \nu_{s}, \nu_{s}\right),
$$

where $\nu:=\left(\nu_{1}, \nu_{2}, \cdots, \nu_{s}\right)$ is a partition of $n$. Now let $\nu=\left(\nu_{1}, \cdots, \nu_{s}\right)$ be a partition of $n$. For any $\mathfrak{t} \in \operatorname{RowStd}(\widetilde{\nu})$, let $d(\mathfrak{t}) \in \mathfrak{S}_{2 n}$ be such that $\mathfrak{t}^{\widetilde{\nu}} d(\mathfrak{t})=\mathfrak{t}$. Let $Y_{\nu, \mathfrak{t}}:=Y_{\nu} \star d(\mathfrak{t})$. For any commutative $\mathbb{Z}$-algebra $R$, we define

$$
\mathcal{M}_{R}^{\lambda}:=R-\operatorname{Span}\left\{Y_{\nu, \mathfrak{t}} \mid \mathfrak{t} \in \operatorname{Std}(\widetilde{\nu}), \lambda \unlhd \nu \in \mathcal{P}_{n}\right\} .
$$

We write $\mathcal{M}^{\lambda}=\mathcal{M}_{\mathbb{Z}}^{\lambda}$. We are interested in the module $\mathcal{M}_{R}^{\lambda}$. In the remaining part of this paper, we shall see that this module is actually a right $\mathfrak{S}_{2 n}$-submodule of $\mathfrak{B}_{n, R}$ (with respect to the action " $\star$ "), and it shares many properties with the permutation module $x_{\widehat{\lambda}} \mathbb{Z}\left[\mathfrak{S}_{2 n}\right]$. In particular, it also has a Specht filtration, and it is stable under base change, i.e., $R \otimes_{\mathbb{Z}} \mathcal{M}^{\lambda} \cong$ $\mathcal{M}_{R}^{\lambda}$ for any commutative $\mathbb{Z}$-algebra $R$.

For our purpose, we need to recall some results in [44] and [42] on the Specht filtrations of permutation modules over the symmetric group $\mathfrak{S}_{2 n}$. Let $\lambda, \mu$ be two partitions of $2 n$. A $\mu$-tableau of type $\lambda$ is a map $\mathrm{S}:[\mu] \rightarrow\{1,2, \cdots, 2 n\}$ such that each $i$ appears exactly $\lambda_{i}$ times. $\mathrm{S}$ is said to be semistandard if each row of $\mathrm{S}$ is nondecreasing and each column of $\mathrm{S}$ is strictly increasing. Let $\mathcal{T}_{0}(\mu, \lambda)$ be the set of all the semistandard $\mu$-tableaux of type $\lambda$. Then $\mathcal{T}_{0}(\mu, \lambda) \neq \emptyset$ only if $\mu \unrhd \lambda$, where " $\unrhd$ " is the dominance order as defined in 44 . For each standard $\mu$-tableau $\mathfrak{s}$, let $\lambda(\mathfrak{s})$ be the tableau which is obtained from $\mathfrak{s}$ by replacing each entry $i$ in $\mathfrak{s}$ by $r$ if $i$ appear in row $r$ of $\mathfrak{t}^{\lambda}$. Then $\lambda(\mathfrak{s})$ is a $\mu$-tableau of type $\lambda$.

For each standard $\mu$-tableau $\mathfrak{t}$ and each semistandard $\mu$-tableau $\mathrm{S}$ of type $\lambda$, we define

$$
x_{\mathrm{S}, \mathfrak{t}}:=\sum_{\mathfrak{s} \in \operatorname{Std}(\mu), \lambda(\mathfrak{s})=\mathrm{S}} d(\mathfrak{s})^{-1} x_{\mu} d(\mathfrak{t}) .
$$

Then by [44, Section 7], the set

$$
\left\{x_{\mathrm{S}, \mathfrak{t}} \mid \mathrm{S} \in \mathcal{T}_{0}(\mu, \lambda), \mathfrak{t} \in \operatorname{Std}(\mu), \lambda \unlhd \mu \vdash 2 n\right\}
$$

form a $\mathbb{Z}$-basis of $x_{\lambda} \mathbb{Z}\left[\mathfrak{S}_{2 n}\right]$. Furthermore, for any commutative $\mathbb{Z}$-algebra $R$, the canonical surjective homomorphism $R \otimes_{\mathbb{Z}} x_{\lambda} \mathbb{Z}\left[\mathfrak{S}_{2 n}\right] \rightarrow x_{\lambda} R\left[\mathfrak{S}_{2 n}\right]$ is an isomorphism.

For each partition $\mu$ of $2 n$ and for each semistandard $\mu$-tableau S of type $\lambda$, according to the results in [44, Section 7] and [42], both the following $\mathbb{Z}$-submodules

$$
\begin{gathered}
M_{\mathrm{S}}^{\lambda}:=\mathbb{Z}-\operatorname{Span}\left(\left\{x_{\mathrm{S}, \mathfrak{s}} \mid \mathfrak{s} \in \operatorname{Std}(\mu)\right\} \bigcup\right. \\
\left.\left\{x_{\mathrm{T}, \mathfrak{t}} \mid \mathrm{T} \in \mathcal{T}_{0}(\nu, \lambda), \mathfrak{t} \in \operatorname{Std}(\nu), \mu \triangleleft \nu \vdash 2 n\right\}\right), \\
M_{\mathrm{S}, \triangleright}^{\lambda}:=\mathbb{Z}-\operatorname{Span}\left\{x_{\mathrm{T}, \mathfrak{t}} \mid \mathrm{T} \in \mathcal{T}_{0}(\nu, \lambda), \mathfrak{t} \in \operatorname{Std}(\nu), \mu \triangleleft \nu \vdash 2 n\right\},
\end{gathered}
$$

are $\mathbb{Z}\left[\mathfrak{S}_{2 n}\right]$-submodules, and the quotient of $M_{\mathrm{S}}^{\lambda}$ by $M_{\mathrm{S}, \triangleright}^{\lambda}$ is canonical isomorphic to $S^{\mu}$ so that the images of the elements $x_{\mathrm{S}, \mathfrak{s}}$, where $\mathfrak{s} \in \operatorname{Std}(\mu)$, form the standard $\mathbb{Z}$-basis of $S^{\mu}$. In this way, it gives rise to a Specht filtration of $x_{\lambda} \mathbb{Z}\left[\mathfrak{S}_{2 n}\right]$. Each semistandard $\mu$-tableau of type $\lambda$ yields a factor which is isomorphic to $S^{\mu}$ so that $x_{\lambda} \mathbb{Z}\left[\mathfrak{S}_{2 n}\right]$ has a series of factors, ordered by $\unlhd$, each isomorphic to some $S^{\mu}, \mu \unrhd \lambda$; moreover, the multiplicity of $S^{\mu}$ is the number of semistandard $\mu$-tableaux of type $\lambda$.

Let $\lambda=\left(\lambda_{1}, \cdots, \lambda_{s}\right)$ be a partition of $n$, where $\lambda_{s}>0$. We write

$$
\widehat{\lambda}=\left(a_{1}^{k_{1}}, a_{2}^{k_{2}}, \cdots, a_{s^{\prime}}^{k_{s^{\prime}}}, a_{s^{\prime}}^{k_{s^{\prime}}}, \cdots, a_{2}^{k_{2}}, a_{1}^{k_{1}}\right),
$$

where $a_{1}>a_{2}>\cdots>a_{s^{\prime}}, k_{i} \in \mathbb{N}$ for each $i, a_{i}^{k_{i}}$ means that $a_{i}$ repeats $k_{i}$ times. Let $\hat{\lambda}^{\prime}$ be the conjugate of $\widehat{\lambda}$. Let $\widetilde{\mathfrak{S}}_{\hat{\lambda}}$ be the subgroup of $\mathfrak{S}_{\widehat{\lambda}^{\prime}}$ consisting of all the elements $w$ satisfying the following condition: for any integers $1 \leq i, j \leq s$ with $\widehat{\lambda}_{i}=\widehat{\lambda}_{j}$, and any integers $a, b$ with 
$1 \leq a, b \leq \widehat{\lambda}_{i}$

$$
\begin{array}{ll} 
& \left(\mathfrak{t}_{\widehat{\lambda}}(i, a)\right) w=\mathfrak{t}_{\widehat{\lambda}}(j, a) \\
\Leftrightarrow & \left.\mathfrak{t}_{\widehat{\lambda}}(i, b)\right) w=\mathfrak{t}_{\widehat{\lambda}}(j, b) \\
\Leftrightarrow & \left.\mathfrak{t}_{\widehat{\lambda}}(2 s+1-i, a)\right) w=\mathfrak{t}_{\widehat{\lambda}}(2 s+1-j, a) \\
\Leftrightarrow & \left.\mathfrak{t}_{\widehat{\lambda}}(2 s+1-i, b)\right) w=\mathfrak{t}_{\widehat{\lambda}}(2 s+1-j, b) .
\end{array}
$$

Let $\widetilde{D}_{\widehat{\lambda}}$ be a complete set of right coset representatives of $\widetilde{\mathfrak{S}}_{\widehat{\lambda}}$ in $\mathfrak{S}_{\widehat{\lambda}^{\prime}}$.

Lemma 6.5. Let $\lambda=\left(\lambda_{1}, \cdots, \lambda_{s}\right)$ be a partition of $n$, where $\lambda_{s}>0$. We keep the notations as above. Let

$$
n_{\widehat{\lambda}}:=\prod_{i=1}^{s^{\prime}} 2^{k_{i}}\left(k_{i} !\right), \quad h_{\widehat{\lambda}}:=\sum_{w \in \widetilde{D}_{\widehat{\lambda}}}(-1)^{\ell(w)} w
$$

Then

$$
Y_{\lambda} \star\left(w_{\widehat{\lambda}} y_{\widehat{\lambda}^{\prime}}\right)=n_{\widehat{\lambda}}\left(Y_{\lambda} \star\left(w_{\widehat{\lambda}} h_{\widehat{\lambda}}\right)\right),
$$

and for any commutative $\mathbb{Z}$-algebra $R, 1_{R} \otimes_{\mathbb{Z}}\left(Y_{\lambda} \star\left(w_{\widehat{\lambda}} h_{\widehat{\lambda}}\right)\right) \neq 0$ in $\mathfrak{B}_{n, R}$.

Proof. By definition,

$$
y_{\widehat{\lambda}^{\prime}}=\sum_{w \in \mathfrak{S}_{\widehat{\lambda}^{\prime}}}(-1)^{\ell(w)} w=\left(\sum_{w \in \widetilde{\mathfrak{S}}_{\widehat{\lambda}}}(-1)^{\ell(w)} w\right) h_{\widehat{\lambda}} .
$$

By definition, it is easy to see that for any $w \in \widetilde{\mathfrak{S}}_{\hat{\lambda}}, \ell(w)$ is an even integer. Now the first statement of this lemma follows from the following identity:

$$
\left(Y_{\lambda} \star w_{\widehat{\lambda}}\right) \star\left(\sum_{w \in \widetilde{\mathfrak{S}}_{\hat{\lambda}^{\prime}}} w\right)=n_{\widehat{\lambda}}\left(Y_{\lambda} \star w_{\widehat{\lambda}}\right) .
$$

Let $d$ be the Brauer $n$-diagram in which the vertex labelled by $\mathfrak{t}_{\widehat{\lambda}}(i, r)$ is connected with the vertex labelled by $\mathfrak{t}_{\hat{\lambda}}(2 s+1-i, r)$ for any $1 \leq i \leq s, 1 \leq r \leq \lambda_{i}$. Then it is easy to see that $d$ appears with coefficient $(-1)^{\ell\left(w_{\widehat{\lambda}}\right)}$ in the expression of $Y_{\lambda} \star\left(w_{\widehat{\lambda}} h_{\widehat{\lambda}}\right)$ as linear combinations of the basis of Brauer $n$-diagrams. It follows that for any commutative $\mathbb{Z}$-algebra $R, 1_{R} \otimes_{\mathbb{Z}}\left(Y_{\lambda} \star\right.$ $\left.\left(w_{\widehat{\lambda}} h_{\widehat{\lambda}}\right)\right) \neq 0$ in $\mathfrak{B}_{n, R}$, as required.

Following $\left[43\right.$, we define the Jucys-Murphy operators of $\mathbb{Z}\left[\mathfrak{S}_{2 n}\right]$.

$$
\left\{\begin{array}{l}
L_{1}:=0 \\
L_{a}:=(a-1, a)+(a-2, a)+\cdots+(1, a), \quad a=2,3, \cdots, 2 n .
\end{array}\right.
$$

Let $\lambda=\left(\lambda_{1}, \cdots, \lambda_{s}\right)$ be a partition of $n$. Then $\widetilde{\lambda}$ is the unique partition obtained by reordering the parts of $\widehat{\lambda}$. Let $z_{\tilde{\lambda}}:=x_{\tilde{\lambda}} w_{\tilde{\lambda}} y_{\widetilde{\lambda}^{\prime}}$. By [11, Lemma 4.3], there is a $\mathbb{Z}\left[\mathfrak{S}_{2 n}\right]$-module isomorphism from $S^{\widehat{\lambda}}$ onto $S^{\widetilde{\lambda}}$, which maps $z_{\widehat{\lambda}}$ to $\pm z_{\widetilde{\lambda}}$. Now applying [12, (3.14)], for each integer $1 \leq a \leq 2 n$, we deduce that

$$
\left(x_{\widehat{\lambda}} w_{\widehat{\lambda}} y_{\widehat{\lambda}^{\prime}}\right) L_{a}=\operatorname{res}_{\mathfrak{t}_{\widehat{\lambda}}}(a)\left(x_{\widehat{\lambda}} w_{\widehat{\lambda}} y_{\widehat{\lambda}^{\prime}}\right) .
$$

For each standard $\tilde{\lambda}$-tableau $\mathfrak{t}$, we define

$$
\Theta_{\mathfrak{t}}:=\prod_{i=1}^{n} \prod_{\substack{\mathfrak{u} \in \operatorname{Std}_{(\widetilde{\lambda})}(\widetilde{\lambda}) \\ \operatorname{res}_{\mathfrak{u}}(i) \neq \operatorname{res}_{\mathfrak{t}}(i)}} \frac{L_{i}-\operatorname{res}_{\mathfrak{u}}(i)}{\operatorname{res}_{\mathfrak{t}}(i)-\operatorname{res}_{\mathfrak{u}}(i)}
$$


By Lemma 6.4 and Frobenius reciprocity, there is a surjective right $\mathbb{Z}\left[\mathfrak{S}_{2 n}\right]$-module homomorphism $\pi_{\lambda}$ from $x_{\widehat{\lambda}} \mathbb{Z}\left[\mathfrak{S}_{2 n}\right]$ onto $Y_{\lambda} \mathbb{Z}\left[\mathfrak{S}_{2 n}\right]$ which extends the map $x_{\widehat{\lambda}} \mapsto Y_{\lambda}$. In particular, by Lemma 6.5.

$$
\left(Y_{\lambda} \star w_{\widehat{\lambda}} h_{\widehat{\lambda}}\right) \star L_{a}=\operatorname{res}_{\mathfrak{t}_{\tilde{\lambda}}}(a)\left(Y_{\lambda} \star\left(w_{\widehat{\lambda}} h_{\widehat{\lambda}}\right)\right) .
$$

Proposition 6.6. Let $\lambda=\left(\lambda_{1}, \cdots, \lambda_{s}\right)$ be a partition of $n$. We have that

$$
\left[Y_{\lambda} \mathbb{Q}\left[\mathfrak{S}_{2 n}\right]: S_{\mathbb{Q}}^{\widetilde{\lambda}}\right]=1 \text {. }
$$

Proof. By Lemma 6.2, we have that

$$
\mathfrak{B}_{n, \mathbb{Q}} \cong \bigoplus_{\mu \in\left(2 \mathcal{P}_{n}\right)^{\prime}} S_{\mathbb{Q}}^{\mu}
$$

It is well-known that each $S_{\mathbb{Q}}^{\mu}$ has a basis $\left\{v_{\mathrm{t}}\right\}_{\mathrm{t} \in \operatorname{Std}(\mu)}$ satisfying

$$
v_{\mathfrak{t}} L_{i}=\operatorname{res}_{\mathfrak{t}}(i) v_{\mathfrak{t}}, \quad \forall 1 \leq i \leq n .
$$

Since $Y_{\lambda} \mathbb{Q}\left[\mathfrak{S}_{2 n}\right] \subseteq \mathfrak{B}_{n, \mathbb{Q}}$, we can write

$$
Y_{\lambda} \star\left(w_{\widehat{\lambda}} h_{\widehat{\lambda}}\right)=\sum_{\mu \in\left(2 \mathcal{P}_{n}\right)^{\prime}} \sum_{\mathfrak{t} \in \operatorname{Std}(\mu)} A_{\mathfrak{t}} v_{\mathfrak{t}}
$$

where $A_{\mathfrak{t}} \in \mathbb{Q}$ for each $\mathfrak{t}$.

For each $\mu \in\left(2 \mathcal{P}_{n}\right)^{\prime}$ and each $\mathfrak{t} \in \operatorname{Std}(\mu)$, we apply the operator $\Theta_{\mathfrak{t}}$ on both sides of the above identity and use Lemma 6.5 and the above discussion. We get that $A_{\mathfrak{t}} \neq 0$ if and only if $\mu=\widetilde{\lambda}$ and $\mathfrak{t}=\mathfrak{t}_{\tilde{\lambda}}$. In other words, $Y_{\lambda} \star\left(w_{\widehat{\lambda}} h_{\widehat{\lambda}}\right)=A_{\mathfrak{t}_{\tilde{\lambda}}} v_{\mathfrak{t}_{\tilde{\lambda}}}$ for some $0 \neq A_{\mathfrak{t}_{\tilde{\lambda}}} \in \mathbb{Q}$. This implies that the projection from $Y_{\lambda} \mathbb{Q}\left[\mathfrak{S}_{2 n}\right]$ to $S_{\mathbb{Q}}^{\widetilde{\lambda}}$ is nonzero. Hence,

$$
\left[Y_{\lambda} \mathbb{Q}\left[\mathfrak{S}_{2 n}\right]: S_{\mathbb{Q}}^{\widetilde{\lambda}}\right]=1
$$

as required.

Suppose $\lambda \in \mathcal{P}_{n}$. Let $\mathcal{D}_{\widehat{\lambda}, \tilde{\lambda}}$ be the set of distinguished $\mathfrak{S}_{\widehat{\lambda}^{-}} \mathfrak{S}_{\widetilde{\lambda}}$ double coset representatives in $\mathfrak{S}_{2 n}$ (cf. [11]). By [13, (1.1)], $d_{\lambda}^{-1} \mathfrak{S}_{\widehat{\lambda}} d_{\lambda}=\mathfrak{S}_{\widetilde{\lambda}}$ for some $d_{\lambda} \in \mathcal{D}_{\widehat{\lambda}, \tilde{\lambda}}$. Hence $x_{\widehat{\lambda}} d_{\lambda}=d_{\lambda} x_{\tilde{\lambda}}$. Then it is easy to see that the set

$$
\left\{d_{\lambda} x_{\mathrm{S}, \mathfrak{t}} \mid \mathrm{S} \in \mathcal{T}_{0}(\mu, \tilde{\lambda}), \mathfrak{t} \in \operatorname{Std}(\mu), \widetilde{\lambda} \unlhd \mu \vdash 2 n\right\}
$$

forms a $\mathbb{Z}$-basis of $x_{\widehat{\lambda}} \mathbb{Z}\left[\mathfrak{S}_{2 n}\right]$, and the sets $d_{\lambda} M_{\mathrm{S}}^{\widetilde{\lambda}}, d_{\lambda} M_{\mathrm{S}, \unrhd}^{\widetilde{\lambda}}$ define Specht filtrations for $x_{\widehat{\lambda}} \mathbb{Z}\left[\mathfrak{S}_{2 n}\right]$.

By the natural surjective right $\mathbb{Z}\left[\mathfrak{S}_{2 n}\right]$-module homomorphism $\pi_{\lambda}$ from $x_{\hat{\lambda}} \mathbb{Z}\left[\mathfrak{S}_{2 n}\right]$ onto $Y_{\lambda} \mathbb{Z}\left[\mathfrak{S}_{2 n}\right]$, we know that the elements $\pi_{\lambda}\left(d_{\lambda} x_{\mathrm{S}, \mathfrak{t}}\right)$, where $\mathrm{S} \in \mathcal{T}_{0}(\mu, \tilde{\lambda}), \mathfrak{t} \in \operatorname{Std}(\mu), \tilde{\lambda} \unlhd \mu \vdash 2 n$, span $Y_{\lambda} \mathbb{Z}\left[\mathfrak{S}_{2 n}\right]$ as $\mathbb{Z}$-module. Recall our definition of $\mathcal{M}^{\lambda}$ in the paragraph below Lemma 6.4.

Proposition 6.7. Let $\lambda$ be as in the previous proposition. For any partition $\mu$ of $2 n$ and any $\mathrm{S} \in \mathcal{T}_{0}(\mu, \widetilde{\lambda})$, we have that $\pi_{\lambda}\left(d_{\lambda} M_{\mathrm{S}}^{\widetilde{\lambda}}\right) \subseteq \mathcal{M}^{\lambda}$. In particular, $Y_{\lambda} \mathbb{Z}\left[\mathfrak{S}_{2 n}\right] \subseteq \mathcal{M}^{\lambda}$.

Proof. We first prove a weak version of the claim in this proposition. That is, for any partition $\mu$ of $2 n$ and any $\mathrm{S} \in \mathcal{T}_{0}(\mu, \widetilde{\lambda})$,

$$
\pi_{\lambda}\left(d_{\lambda} M_{\mathrm{S}}^{\widetilde{\lambda}}\right) \subseteq \mathcal{M}_{\mathbb{Q}}^{\lambda}
$$

We consider the dominance order " $\unlhd$ " and make induction on $\lambda$. We start with the partition $\lambda=(n)$, which is the unique maximal partition of $n$ with respect to " $\unlhd$ ". Then $\widehat{\lambda}=(n, n)=\widetilde{\lambda}$ 
and $d_{\lambda}=1$. Let

$$
S:=\left(\begin{array}{l}
1,1, \cdots, 1 \\
2,2, \cdots, 2
\end{array}\right)
$$

be the unique semistandard $(n, n)$-tableau in $\mathcal{T}_{0}((n, n),(n, n))$. Since $\mathbb{Q} \otimes_{\mathbb{Z}} M_{\mathrm{S}, \triangleright}^{\tilde{\lambda}}$ contains no composition factors in $\left\{S_{\mathbb{Q}}^{\widetilde{\nu}} \mid \nu \in\left(2 \mathcal{P}_{n}\right)^{\prime}\right\}$, it follows that $\pi_{\lambda}\left(M_{\mathrm{S}, \triangleright}^{\widetilde{\lambda}}\right)=0$. Hence $\pi_{\lambda}$ induces a surjective homomorphism

$$
S_{\mathbb{Q}}^{(n, n)} \cong M_{\mathrm{S}}^{\widetilde{\lambda}} / M_{\mathrm{S}, \triangleright}^{\widetilde{\lambda}} \rightarrow Y_{\lambda} \mathbb{Q}\left[\mathfrak{S}_{2 n}\right],
$$

by which it is easy to see the claim in this proposition is true for $\lambda=(n)$.

Now let $\lambda \triangleleft(n)$ be a partition of $n$. Assume that for any partition $\nu$ of $n$ satisfying $\nu \triangleright \lambda$, the claim in this proposition is true. We now prove the claim for the partition $\lambda$.

Let $\mu \triangleright \lambda$ be a partition of $2 n$ with $\mathcal{T}_{0}(\mu, \widetilde{\lambda}) \neq \emptyset$. We consider again the dominance order " $\unlhd$ " and make induction on $\mu$. Since $\mathcal{T}_{0}((2 n), \tilde{\lambda})$ contains a unique element $S_{\star}, \operatorname{Std}((2 n))=\left\{\mathfrak{t}^{(2 n)}\right\}$, by Lemma 6.2, it is clear that

$$
\pi_{\lambda}\left(d_{\lambda} x_{\mathrm{S}_{*}, \mathrm{t}^{(2 n)}}\right)=\pi_{\lambda}\left(x_{(2 n)}\right)=0 \in \mathcal{M}^{\lambda} .
$$

So in this case the claim of this proposition is still true.

Now let $\mu \triangleright \widetilde{\lambda}$ be a partition of $2 n$ with $\mathcal{T}_{0}(\mu, \widetilde{\lambda}) \neq \emptyset$ and $\mu \triangleleft(2 n)$. Assume that for any partition $\nu$ of $2 n$ satisfying $\mathcal{T}_{0}(\nu, \tilde{\lambda}) \neq \emptyset$ and $\nu \triangleright \mu$,

$$
\pi_{\lambda}\left(d_{\lambda} M_{\mathrm{S}}^{\widetilde{\lambda}}\right) \subseteq \mathcal{M}_{\mathbb{Q}}^{\lambda}
$$

for any $S \in \mathcal{T}_{0}(\nu, \widetilde{\lambda})$.

Let $\mathrm{S} \in \mathcal{T}_{0}(\mu, \tilde{\lambda})$. The homomorphism $\pi_{\lambda}$ induces a surjective map from $d_{\lambda} M_{\mathrm{S}}^{\tilde{\lambda}} / d_{\lambda} M_{\mathrm{S}, \triangleright}^{\tilde{\lambda}}$ onto

$$
\left(\pi_{\lambda}\left(d_{\lambda} M_{\mathrm{S}}^{\tilde{\lambda}}\right)\right) /\left(\pi_{\lambda}\left(d_{\lambda} M_{\mathrm{S}, \triangleright}^{\tilde{\lambda}}\right)\right) .
$$

Hence it also induces a surjective map $\widetilde{\pi}_{\lambda}$ from

$$
\left(\mathbb{Q} \otimes_{\mathbb{Z}} d_{\lambda} M_{\mathrm{S}}^{\tilde{\lambda}} /\left(\mathbb{Q} \otimes_{\mathbb{Z}} d_{\lambda} M_{\mathrm{S}, \triangleright}^{\tilde{\lambda}}\right) \cong \mathbb{Q} \otimes_{\mathbb{Z}}\left(d_{\lambda} M_{\mathrm{S}}^{\tilde{\lambda}} / d_{\lambda} M_{\mathrm{S}, \triangleright}^{\tilde{\lambda}}\right) \cong S_{\mathbb{Q}}^{\mu}\right.
$$

onto

$$
\mathbb{Q} \otimes_{\mathbb{Z}}\left(\pi_{\lambda}\left(d_{\lambda} M_{\mathrm{S}}^{\widetilde{\lambda}}\right) / \pi_{\lambda}\left(d_{\lambda} M_{\mathrm{S}, \triangleright}^{\tilde{\lambda}}\right)\right) .
$$

Since $S_{\mathbb{Q}}^{\mu}$ is irreducible, the above map is either a zero map or an isomorphism. If it is a zero map, then (by induction hypothesis)

$$
\pi_{\lambda}\left(d_{\lambda} M_{\mathrm{S}}^{\widetilde{\lambda}}\right) \subseteq \pi_{\lambda}\left(d_{\lambda} M_{\mathrm{S}, \triangleright}^{\widetilde{\lambda}}\right) \subseteq \mathcal{M}_{\mathbb{Q}}^{\lambda} .
$$

It remains to consider the case where $\widetilde{\pi}_{\lambda}$ is an isomorphism. In particular,

$$
\mathbb{Q} \otimes_{\mathbb{Z}}\left(\pi_{\lambda}\left(d_{\lambda} M_{\mathrm{S}}^{\tilde{\lambda}}\right) / \pi_{\lambda}\left(d_{\lambda} M_{\mathrm{S}, \triangleright}^{\tilde{\lambda}}\right)\right) \cong S_{\mathbb{Q}}^{\mu}
$$

Applying Lemma 6.2, we know that $\mu \in\left(2 \mathcal{P}_{n}\right)^{\prime}$. Therefore we can write $\mu=\widetilde{\nu}$ for some $\nu \in \mathcal{P}_{n}$. Note that $\mu \triangleright \widetilde{\lambda}$ implies that $\nu \triangleright \lambda$.

On the other hand, there is also a surjective homomorphism $\pi_{\nu}$ from $x_{\widehat{\nu}} \mathbb{Z}\left[\mathfrak{S}_{2 n}\right] / d_{\nu} M_{\mathrm{S}_{0}, \triangleright}^{\widetilde{\nu}}$ onto

$$
\left(\pi_{\nu}\left(x_{\widehat{\nu}} \mathbb{Z}\left[\mathfrak{S}_{2 n}\right]\right)\right) /\left(\pi_{\nu}\left(d_{\nu} M_{\mathrm{S}_{0}, \triangleright}^{\widetilde{\nu}}\right)\right)=Y_{\nu} \mathbb{Z}\left[\mathfrak{S}_{2 n}\right] /\left(\pi_{\nu}\left(d_{\nu} M_{\mathrm{S}_{0}, \triangleright}^{\widetilde{\nu}}\right)\right),
$$

where $\mathrm{S}_{0}$ is the unique semistandard $\mu$-tableau in $\mathcal{T}_{0}(\mu, \widetilde{\nu})$ in which the numbers $1,1, \cdots, 1,2,2, \cdots, 2, \cdots, 2 n, \cdots, 2 n$ appears in order along successive rows. Hence it also induces a surjective map $\tilde{\pi}_{\nu}$ from

$$
\left(\mathbb{Q} \otimes_{\mathbb{Z}} x_{\widehat{\nu}} \mathbb{Z}\left[\mathfrak{S}_{2 n}\right] /\left(\mathbb{Q} \otimes_{\mathbb{Z}} d_{\nu} M_{\mathrm{S}_{0}, \triangleright}^{\widetilde{\nu}}\right) \cong \mathbb{Q} \otimes_{\mathbb{Z}}\left(x_{\widehat{\nu}} \mathbb{Z}\left[\mathfrak{S}_{2 n}\right] / d_{\nu} M_{\mathrm{S}_{0}, \triangleright}^{\widetilde{\nu}}\right) \cong S_{\mathbb{Q}}^{\mu}\right.
$$


onto

$$
\mathbb{Q} \otimes_{\mathbb{Z}}\left(Y_{\nu} \mathbb{Z}\left[\mathfrak{S}_{2 n}\right] / \pi_{\nu}\left(d_{\nu} M_{\mathrm{S}_{0}, \triangleright}^{\widetilde{\nu}}\right)\right) \cong\left(\mathbb{Q} \otimes_{\mathbb{Z}} Y_{\nu} \mathbb{Z}\left[\mathfrak{S}_{2 n}\right]\right) /\left(\mathbb{Q} \otimes_{\mathbb{Z}} \pi_{\nu}\left(d_{\nu} M_{\mathrm{S}_{0}, \triangleright}^{\widetilde{\nu}}\right)\right)
$$

It is well-known that $S_{\mathbb{Q}}^{\mu}$ does not occur as composition factor in $\mathbb{Q} \otimes_{\mathbb{Z}} d_{\nu} M_{\mathrm{S}_{0}, \triangleright}^{\widetilde{\nu}}$. Hence $S_{\mathbb{Q}}^{\mu}$ does not occur as composition factor in

$$
\mathbb{Q} \otimes_{\mathbb{Z}} \pi_{\nu}\left(d_{\nu} M_{\mathrm{S}_{0}, \triangleright}^{\widetilde{\nu}}\right) .
$$

By Proposition [6.6] $S_{\mathbb{Q}}^{\mu}$ occurs as composition factor with multiplicity one in $\mathbb{Q} \otimes_{\mathbb{Z}} Y_{\nu} \mathbb{Z}\left[\mathfrak{S}_{2 n}\right]$. Therefore,

$$
\mathbb{Q} \otimes_{\mathbb{Z}} Y_{\nu} \mathbb{Z}\left[\mathfrak{S}_{2 n}\right] \neq \mathbb{Q} \otimes_{\mathbb{Z}} \pi_{\nu}\left(d_{\nu} M_{\mathrm{S}_{0}, \triangleright}^{\widetilde{\nu}}\right)
$$

It follows that $\widetilde{\pi}_{\nu}$ must be an isomorphism. Hence

$$
\mathbb{Q} \otimes_{\mathbb{Z}}\left(Y_{\nu} \mathbb{Z}\left[\mathfrak{S}_{2 n}\right] / \pi_{\nu}\left(d_{\nu} M_{\mathrm{S}_{0}, \triangleright}^{\widetilde{\nu}}\right)\right) \cong S_{\mathbb{Q}}^{\mu} .
$$

We write $A=\pi_{\lambda}\left(d_{\lambda} M_{\mathrm{S}}^{\widetilde{\lambda}}\right), B=Y_{\nu} \mathbb{Z}\left[\mathfrak{S}_{2 n}\right]$. Since $S_{\mathbb{Q}}^{\mu}$ appears only once in $\mathfrak{B}_{n, \mathbb{Q}}$, it follows that $S_{\mathbb{Q}}^{\mu}$ must occur as composition factor in the module

$$
\left(\mathbb{Q} \otimes_{\mathbb{Z}} A\right) \cap\left(\mathbb{Q} \otimes_{\mathbb{Z}} B\right)=\mathbb{Q} \otimes_{\mathbb{Z}}(A \cap B) .
$$

Hence $S_{\mathbb{Q}}^{\mu}$ can not occur as composition factor in the module

$$
\left(\mathbb{Q} \otimes_{\mathbb{Z}} A\right) /\left(\mathbb{Q} \otimes_{\mathbb{Z}}(A \cap B)\right) \cong \mathbb{Q} \otimes_{\mathbb{Z}}(A / A \cap B) .
$$

Therefore, the image of the canonical projection $\mathbb{Q} \otimes_{\mathbb{Z}} A \rightarrow \mathbb{Q} \otimes_{\mathbb{Z}}(A / A \cap B)$ must be contained in the image of $\mathbb{Q} \otimes_{\mathbb{Z}} \pi_{\lambda}\left(d_{\lambda} M_{\mathrm{S}, \triangleright}^{\widetilde{\lambda}}\right)$. However, by induction hypothesis, both $\pi_{\lambda}\left(d_{\lambda} M_{\mathrm{S}, \triangleright}^{\widetilde{\lambda}}\right)$ and $B$ are contained in the $\mathbb{Q}$-span of $\left\{Y_{\alpha, \mathfrak{u}} \mid \mathfrak{u} \in \operatorname{Std}(\widetilde{\alpha}), \lambda \unlhd \alpha \in \mathcal{P}_{n}\right\}$. It follows that

$$
\pi_{\lambda}\left(d_{\lambda} M_{\mathrm{S}}^{\widetilde{\lambda}}\right) \subseteq \mathcal{M}_{\mathbb{Q}}^{\lambda},
$$

as required.

Suppose that

$$
\pi_{\lambda}\left(d_{\lambda} M_{\mathrm{S}}^{\widetilde{\lambda}}\right) \nsubseteq \mathcal{M}^{\lambda}
$$

Then (by the $\mathbb{Z}$-freeness of $\mathfrak{B}_{n}$ ) there exists an element $x \in d_{\lambda} M_{\mathrm{S}}^{\widetilde{\lambda}}$, integers $a, a_{\mathfrak{u}}$, and a prime divisor $p \in \mathbb{N}$ of $a$, such that

$$
a \pi_{\lambda}(x)=\sum_{\lambda \unlhd \alpha \in \mathcal{P}_{n}} \sum_{\mathfrak{u} \in \operatorname{Std}(\widetilde{\alpha})} a_{\mathfrak{u}} Y_{\alpha} \star d(\mathfrak{u}),
$$

and $\Sigma_{p}:=\left\{\alpha \in \mathcal{P}_{n} \mid \lambda \unlhd \alpha, p \nmid a_{\mathfrak{u}}\right.$, for some $\left.\mathfrak{u} \in \operatorname{Std}(\widetilde{\alpha})\right\} \neq \emptyset$.

We take an $\alpha \in \Sigma_{p}$ such that $\alpha$ is minimal with respect to " $\unlhd$ ". Then we take an $u \in \operatorname{Std}(\widetilde{\alpha})$ such that $p \nmid a_{\mathfrak{u}}$ and $\ell(d(\mathfrak{u}))$ is maximal among the elements in the set $\left\{\mathfrak{u} \in \operatorname{Std}(\widetilde{\alpha}) \mid p \nmid a_{\mathfrak{u}}\right\}$. Let $\sigma_{\mathfrak{u}}$ be the unique element in $\mathfrak{S}_{2 n}$ such that $d(\mathfrak{u}) \sigma_{\mathfrak{u}}=w_{\alpha}$ and $\ell\left(w_{\alpha}\right)=\ell(d(\mathfrak{u}))+\ell\left(\sigma_{\mathfrak{u}}\right)$. We consider the finite field $\mathbb{F}_{p}$ as a $\mathbb{Z}$-algebra. By [11, (4.1)], we know that for any composition $\beta$ of $2 n$, any $\gamma \in \mathcal{P}_{2 n}$, and element $w \in \mathfrak{S}_{2 n}$,

$$
x_{\beta} w y_{\gamma^{\prime}} \neq 0 \text { only if } \gamma \unrhd \beta \text {; while } x_{\beta} w y_{\beta^{\prime}} \neq 0 \text { only if } w \in \mathfrak{S}_{\beta} w_{\beta} \text {. }
$$

Hence for any $\beta \in \mathcal{P}_{n}, \gamma \in \mathcal{P}_{2 n}$,

$$
Y_{\beta} \star\left(w y_{\gamma^{\prime}}\right) \neq 0 \text { only if } \gamma \unrhd \widehat{\beta} ; Y_{\beta} \star\left(w y_{\widehat{\beta}^{\prime}}\right) \neq 0 \text { only if } w \in \mathfrak{S}_{\widehat{\beta}} w_{\widehat{\beta}} .
$$

Now applying Lemma 6.5, we get

$$
0=1_{\mathbb{F}_{p}} \otimes_{\mathbb{Z}}\left(a \pi_{\lambda}(x) \star\left(\sigma_{\mathfrak{u}} h_{\widehat{\alpha}}\right)\right)= \pm 1_{\mathbb{F}_{p}} \otimes_{\mathbb{Z}}\left(a_{\mathfrak{u}} Y_{\alpha} \star\left(w_{\widehat{\alpha}} h_{\widehat{\alpha}}\right)\right) \neq 0,
$$

which is a contradiction. This proves that $\pi_{\lambda}\left(d_{\lambda} M_{\mathrm{S}}^{\widetilde{\lambda}}\right) \subseteq \mathcal{M}^{\lambda}$. 
Corollary 6.8. For any partition $\lambda \in \mathcal{P}_{n}$ and any commutative $\mathbb{Z}$-algebra $R, \mathcal{M}_{R}^{\lambda}$ is a right $\mathfrak{S}_{2 n}$-submodule of $\mathfrak{B}_{n, R}$.

Proof. This follows directly from Proposition 6.7.

Theorem 6.9. For any partition $\lambda \in \mathcal{P}_{n}$ and any commutative $\mathbb{Z}$-algebra $R$, the canonical map $R \otimes_{\mathbb{Z}} \mathcal{M}^{\lambda} \rightarrow \mathcal{M}_{R}^{\lambda}$ is an isomorphism, and the set

$$
\left\{Y_{\nu, \mathfrak{t}} \mid \mathfrak{t} \in \operatorname{Std}(\widetilde{\nu}), \lambda \unlhd \nu \in \mathcal{P}_{n}\right\}
$$

forms an $R$-basis of $\mathcal{M}_{R}^{\lambda}$. In particular, the set

$$
\left\{Y_{\lambda, \mathfrak{t}} \mid \mathfrak{t} \in \operatorname{Std}(\widetilde{\lambda}), \lambda \in \mathcal{P}_{n}\right\}
$$

forms an $R$-basis of $\mathfrak{B}_{n, R}$.

Proof. We take $\lambda=\left(1^{n}\right)$, then $Y_{\lambda} \mathbb{Z}\left[\mathfrak{S}_{2 n}\right]=\mathfrak{B}_{n}$. It is well-known that $\mathfrak{B}_{n, R} \cong R \otimes_{\mathbb{Z}} \mathfrak{B}_{n}$ for any commutative $\mathbb{Z}$-algebra $R$. Applying Proposition 6.7, we get that for any commutative $\mathbb{Z}$-algebra $R$, the set

$$
\left\{Y_{\lambda, \mathfrak{t}} \mid \mathfrak{t} \in \operatorname{Std}(\widetilde{\lambda}), \lambda \in \mathcal{P}_{n}\right\}
$$

must form an $R$-basis of $\mathfrak{B}_{n, R}$. By the $R$-linear independence of the elements in this set and Corollary 6.8. we also get that, for any partition $\lambda \in 2 \mathcal{P}_{n}$, the set

$$
\left\{Y_{\nu, \mathfrak{t}} \mid \mathfrak{t} \in \operatorname{Std}(\widetilde{\nu}), \lambda \unlhd \nu \in \mathcal{P}_{n}\right\}
$$

must form an $R$-basis of $\mathcal{M}_{R}^{\lambda}$. Therefore, for any commutative $\mathbb{Z}$-algebra $R$, the canonical map $R \otimes_{\mathbb{Z}} \mathcal{M}^{\lambda} \rightarrow \mathcal{M}_{R}^{\lambda}$ is an isomorphism.

Theorem 6.10. For any partition $\lambda \in \mathcal{P}_{n}$ and any commutative $\mathbb{Z}$-algebra $R$, we define

$$
\mathcal{M}_{R}^{\triangleright \lambda}:=R-\operatorname{Span}\left\{Y_{\nu, \mathfrak{t}} \mid \mathfrak{t} \in \operatorname{Std}(\widetilde{\nu}), \lambda \triangleleft \nu \in \mathcal{P}_{n}\right\} .
$$

Then $\mathcal{M}_{R}^{\triangleright \lambda}$ is a right $R\left[\mathfrak{S}_{2 n}\right]$-submodule of $\mathcal{M}_{R}^{\lambda}$, and there is a $R\left[\mathfrak{S}_{2 n}\right]$-module isomorphism

$$
\mathcal{M}_{R}^{\lambda} / \mathcal{M}_{R}^{\triangleright \lambda} \cong S_{R}^{\tilde{\lambda}}
$$

In particular, $\mathfrak{B}_{n, R}$ has a Specht filtration.

Proof. It suffices to consider the case where $R=\mathbb{Z}$. We first show that

$$
\mathcal{M}_{\mathbb{Q}}^{\lambda} \cong \oplus_{\lambda \unlhd \mu \in \mathcal{P}_{n}} S_{\mathbb{Q}}^{\widetilde{\mu}}, \quad \mathcal{M}_{\mathbb{Q}}^{\triangleright \lambda} \cong \oplus_{\lambda \triangleleft \mu \in \mathcal{P}_{n}} S_{\mathbb{Q}}^{\widetilde{\mu}}
$$

For each $\mu \in \mathcal{P}_{n}$, we use $\rho_{\mu}^{\lambda}$ to denote the composite of the embedding $\mathcal{M}_{\mathbb{Q}}^{\lambda} \hookrightarrow \mathfrak{B}_{n, \mathbb{Q}}$ and the projection $\mathfrak{B}_{n, \mathbb{Q}} \rightarrow S_{\mathbb{Q}}^{\widetilde{\mu}}$. Suppose that $\rho_{\mu}^{\lambda} \neq 0$. Then $\rho_{\mu}^{\lambda}$ must be a surjection. We claim that $\mu \unrhd \lambda$. In fact, if $\mu \unrhd \lambda$, then for any $\lambda \unlhd \nu \in \mathcal{P}_{n}, \mu \nsubseteq \nu$, and $x_{\widehat{\nu}} \mathbb{Z}\left[\mathfrak{S}_{2 n}\right] w_{\widehat{\mu}^{\prime}} x_{\widehat{\mu}} w_{\widehat{\mu}} y_{\widehat{\mu}^{\prime}}=0$, hence $Y_{\nu, \mathfrak{t}} \star\left(w_{\widehat{\mu}^{\prime}} x_{\widehat{\mu}} w_{\widehat{\mu}} y_{\widehat{\mu}^{\prime}}\right)=0$ for any $\mathfrak{t} \in \operatorname{Std}(\widetilde{\nu})$. It follows that $\mathcal{M}_{\mathbb{Q}}^{\lambda}\left(w_{\widehat{\mu}^{\prime}} x_{\widehat{\mu}} w_{\widehat{\mu}} y_{\widehat{\mu}^{\prime}}\right)=0$. Therefore, $S_{\mathbb{Q}}^{\widetilde{\mu}}\left(w_{\mu^{\prime}} x_{\widehat{\mu}} w_{\widehat{\mu}} y_{\widehat{\mu}^{\prime}}\right)=0$. On the other hand, since $S_{\mathbb{Q}}^{\widetilde{\mu}} \cong x_{\widehat{\mu}} w_{\widehat{\mu}} y_{\widehat{\mu}^{\prime}} \mathbb{Q}\left[\mathfrak{S}_{2 n}\right]$, and by [36. Lemma 5.7],

$$
x_{\widehat{\mu}} w_{\widehat{\mu}} y_{\widehat{\mu}^{\prime}}\left(w_{\widehat{\mu}^{\prime}} x_{\widehat{\mu}} w_{\widehat{\mu}} y_{\widehat{\mu}^{\prime}}\right)=\left(\prod_{(i, j) \in[\widehat{\mu}]} h_{i, j}^{\widehat{\mu}}\right) x_{\widehat{\mu}} w_{\widehat{\mu}} y_{\widehat{\mu}^{\prime}} \neq 0,
$$


where $h_{i, j}^{\widehat{\mu}}$ is the $(i, j)$-hook length in $[\widehat{\mu}]$, we get a contradiction. Therefore, $\rho_{\mu}^{\lambda} \neq 0$ must imply that $\mu \unrhd \lambda$. Now counting the dimensions, we deduce that $\mathcal{M}_{\mathbb{Q}}^{\lambda} \cong \oplus_{\lambda \unlhd \mu \in \mathcal{P}_{n}} S_{\mathbb{Q}}^{\widetilde{\mu}}$. In a similar way, we can prove that $\mathcal{M}_{\mathbb{Q}}^{\triangleright \lambda} \cong \oplus_{\lambda \triangleleft \mu \in \mathcal{P}_{n}} S_{\mathbb{Q}}^{\widetilde{\mu}}$. It follows that $\mathcal{M}_{\mathbb{Q}}^{\lambda} / \mathcal{M}_{\mathbb{Q}}^{\triangleright \lambda} \cong S_{\mathbb{Q}}^{\widetilde{\lambda}}$.

We now consider the natural map from $x_{\widehat{\lambda}} \mathbb{Z}\left[\mathfrak{S}_{2 n}\right]$ onto $\mathcal{M}^{\lambda} / \mathcal{M}^{\triangleright \lambda}$. Since $\mathbb{Q} \otimes_{\mathbb{Z}} d_{\lambda} M_{\mathrm{S}_{0}, \triangleright}^{\widetilde{\lambda}}$ does not contain $S_{\mathbb{Q}}^{\tilde{\lambda}}$ as a composition factor, it follows that (by Proposition 6.7) the image of $d_{\lambda} M_{\mathrm{S}_{0}, \triangleright}^{\widetilde{\lambda}}$ must be 0 . Therefore we get a surjective map from $S^{\widetilde{\lambda}}$ onto $\mathcal{M}^{\lambda} / \mathcal{M}^{\triangleright \lambda}$. This map sends the standard basis of $S^{\widetilde{\lambda}}$ to the canonical basis of $\mathcal{M}^{\lambda} / \mathcal{M}^{\triangleright \lambda}$. So it must be injective as well, as required.

\section{The second main result}

In this section, we shall use Theorem 1.2 and the results obtained in Section 6 to give an explicit and characteristic-free description of the annihilator of the $n$-tensor space $V^{\otimes n}$ in the Brauer algebra $\mathfrak{B}_{n}(m)$.

Let $K$ be an arbitrary infinite field of odd characteristic. Let $m, n \in \mathbb{N}$. Let $V$ be the $m$ dimensional orthogonal $K$-vector space we introduced before. Let $O(V)$ be the corresponding orthogonal group, acting naturally on $V$, and hence on the $n$-tensor space $V^{\otimes n}$ from the lefthand side. As we mentioned in the introduction, this left action on $V^{\otimes n}$ is centralized by the specialized Brauer algebra $\mathfrak{B}_{n}(m)_{K}:=K \otimes_{\mathbb{Z}} \mathfrak{B}_{n}(m)$, where $K$ is regarded as $\mathbb{Z}$-algebra in a natural way. The Brauer algebra $\mathfrak{B}_{n}(m)_{K}$ acts on $n$-tensor space $V^{\otimes n}$ from the right-hand side. Let $\varphi$ be the natural $K$-algebra homomorphism

$$
\varphi:\left(\mathfrak{B}_{n}(m)_{K}\right)^{\text {op }} \rightarrow \operatorname{End}_{K}\left(V^{\otimes n}\right) .
$$

Recall (see (5.4) ) that there is an isomorphism $\operatorname{End}_{K}\left(V^{\otimes n}\right) \cong\left(V^{\otimes 2 n}\right)^{*}$, and the place permutation action of the symmetric group $\mathfrak{S}_{2 n}$ on $V^{\otimes 2 n}$ naturally induces an action on $\operatorname{End}_{K}\left(V^{\otimes n}\right) \cong\left(V^{\otimes 2 n}\right)^{*}$. By Lemma 5.5. we know that $\varphi$ is a $\mathfrak{S}_{2 n}$-module homomorphism. Using the $O_{m}(\mathbb{C})-\mathbb{C S}_{2 n}$-bimodule decomposition of $V_{\mathbb{C}}^{\otimes 2 n}$ (cf. [38]), it is easy to check that

$$
\operatorname{dim}\left(V_{\mathbb{C}}^{\otimes 2 n}\right)^{O_{m}(\mathbb{C})}=\sum_{\substack{\lambda \in\left(2 \mathcal{P}_{n}\right)^{\prime} \\ \lambda_{1} \leq m}} \operatorname{dim} S^{\lambda} .
$$

Now applying Theorem 1.2, Lemma 3.7 and our previous discussion, we deduce that

LEMma 7.1. With the notations as above, we have that

$$
\operatorname{dim}(\operatorname{Ker} \varphi)=\sum_{\substack{\lambda \in\left(2 \mathcal{P}_{n}\right)^{\prime} \\ \lambda_{1}>m}} \operatorname{dim} S^{\lambda} .
$$

We remark that when $K=\mathbb{C}$, the above result was deduced in the work of $[\mathbf{3 4}$ and $2 \mathbf{2 3}$ Proposition 1.6].

Proof of Theorem 1.4; For any $\lambda \in \mathcal{P}_{n}$, it is easy to see that $\lambda \unrhd\left(m+1,1^{n-m-1}\right)$ if and only if $\lambda_{1}>m$. Therefore, by Theorem 6.9 and Lemma 7.1, $\operatorname{dim} \operatorname{Ker} \varphi=\operatorname{dim} \mathcal{M}_{K}^{\left(m+1,1^{n-m-1}\right)}$. 
Furthermore, by Lemma 7.1 Ker $\varphi$ is a $\mathfrak{S}_{2 n}$-submodule of $\mathfrak{B}_{n}(m)$. Therefore, by Lemma 7.1 , to prove the theorem, it suffices to show that $Y_{\lambda} \in \operatorname{Ker} \varphi$ for each partition $\lambda \in \mathcal{P}_{n}$ satisfying $\lambda_{1}>m$.

By definition, $Y_{\lambda}:=Y_{\left(\lambda_{1}\right)}^{(0)} Y_{\left(\lambda_{2}\right)}^{\left(\lambda_{1}\right)} \cdots Y_{\left(\lambda_{s}\right)}^{\left(\lambda_{1}+\lambda_{2}+\cdots+\lambda_{s-1}\right)}$. By [28, we know that $Y_{\left(\lambda_{1}\right)}^{(0)} \in \operatorname{Ker} \varphi$

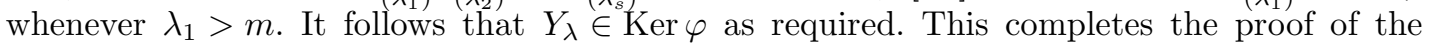
theorem.

\section{References}

1. A.M. AdAmovich and G.L. Rybnikov, 'Tilting modules for classical groups and Howe duality in positive charactersitic', Transformation groups 1 (1996) 1-33.

2. M.F. ATiYAh and I.G. Macdonald, Introduction to commutative algebra (Addison-Wesley, 1969).

3. R. Brauer, 'On algebras which are connected with semisimple continuous groups', Ann. of Math. 38 (1937) $857-872$.

4. W. P. Brown, 'An algebra related to the orthogonal group', Michigan Math. J. 3 (1955-1956) 1-22.

5. W. P. Brown, 'The semisimplicity of $\omega_{f}^{n}$ ', Ann. of Math. 63 (1956) 324-335.

6. G. ClifF, 'A basis of bideterminants for the coordinate ring of the orthogonal group', Communications in Algebra (7) 36 (2008) 2719-2749.

7. A. Cox, M. De Visscher and P. Martin, 'The blocks of the Brauer algebra in characteristic zero', Preprint, 2006, arXiv:math.RT/0601387

8. R. W. CARTER and G. LUSZTiG, 'On the modular representations of general linear and symmetric groups', Math. Z. 136 (1974) 193-242.

9. M. Demazure and P. Gabriel, Introduction to algebraic geometry and algebraic groups (North-Holland, Amsterdam, 1970).

10. R. Dipper, S. Doty and J. Hu, 'Brauer algebras, symplectic Schur algebras and Schur-Weyl duality', Trans. Amer. Math. Soc. 360 (2008) 189-213.

11. R. Dipper and G. D. James, 'Representations of Hecke algebras of general linear groups', Proc. London. Math. Soc. (3) 52 (1986) 20-52.

12. R. Dipper and G. D. James, 'Blocks and idempotents of Hecke algebras of general linear groups', Proc. London. Math. Soc. (3) 54 (1987) 57-82.

13. R. Dipper and G. D. James, ' $q$-tensor space and $q$-Weyl modules', Trans. Amer. Math. Soc. 327 (1991) $251-282$.

14. S. Donkin, Rational representations of algebraic groups, Lect. Notes in Math. Vol. 1140 (Springer-Verlag, 1985).

15. S. Donkin, 'On Schur algebras and related algebras I', J. Algebra 104 (1986) 310-328.

16. S. Donkin, 'On Schur algebras and related algebras II', J. Algebra 111 (1987) 354-364.

17. C. De Concini and C. Procesi, 'A characteristic free approach to invariant theory', Adv. Math. 21 (1976) $330-354$.

18. S. Dоту, 'Polynomial representations, algebraic monoids, and Schur algebras of classical type', J. Pure Appl. Algebra 123 (1998) 165-199.

19. S. Doty, 'Representation theory of reductive normal algebraic monoids', Trans. Amer. math. Soc. 351 (1999) 2539-2551.

20. D. Eisenbud, Commutative algebra, with a view toward algebraic geometry, Graduate Texts in Mathematics, 150 (Springer-Verlag, 1994).

21. J. Enyang, 'Cellular bases for the Brauer and Birman-Murakami-Wenzl algebras', J. Algebra 281 (2004) 413-449.

22. S. Fishel and I. Grojnowski, 'Canonical bases for the Brauer centralizer algebra', Math. Res. Lett. (1) 2 (1995) 15-26.

23. F. Gavarini, 'A Brauer algebra theoretic proof of Littlewood's restriction rules', J. Algebra 212 (1999) 240-271.

24. F. Gavarini, 'On the radical of Brauer algebras', Math. Zeit. 260 (2008) 673-697.

25. J. J. Graham and G. I. Lehrer, 'Cellular algebras', Invent. Math. 123 (1996) 1-34.

26. R. Goodman and N. R. WALLACH, Representations and invariants of classical groups (Cambridge University Press, 1998).

27. J. A. Green, Polynomial representations of $G L_{n}$, Lect. Notes in Math. Vol. 830 (Springer-Verlag, 1980).

28. M. Härterich, 'Murphy bases of generalized Temperley-Lieb algebras', Arch. Math. (5) 72 (1999) 337-345.

29. J. Hu, 'Quasi-parabolic subgroups of the Weyl group of type D', European Journal of Combinatorics, (3) 28 (2007) 807-821. 
30. J. Hu, 'Specht filtrations and tensor spaces for the Brauer algebra', J. Algebr. Comb., (2) 28 (2008), 281-312.

31. P. HAnlon and D. B. WAles, 'On the decomposition of Brauer's centralizer algebras', J. Algebra 121 (1989) 409-445.

32. P. HANlON and D. B. WAles, 'Eigenvalues connected with Brauer's centralizer algebras', J. Algebra 121 (1989) $446-475$.

33. J. C. Jantzen, Representations of Algebraic Groups, second edition (American Mathematical Society, 2003).

34. J.-L. LodAy and C. Procesi, 'Homology of symplectic and orthogonal algebras', Adv. Math. 21 (1988) 93-108.

35. G. LuszTig, Introduction to Quantum Groups, Progress in Math., 110 (Birkhäuser, Boston, 1990).

36. M. Künzer and A. MAthas, 'Elementary divisors of Specht modules', European J. Combinatorics, 26 (2005) 943-964.

37. S. KÖNig, I.H. SlungÅRd and C. XI, 'Double centralizer properties, dominant dimension, and tilting modules', J. Algebra 240 (2001) 393-412.

38. K. KoIKe and I. TeradA, 'Young-diagrammatic methods for the representation theory of the classical groups of type $B_{n}, C_{n}, D_{n}$ ', J. Algebra 107 (1987) 466-511.

39. Q. Liu, 'Schur algebras of classical groups', J. Algebra 301 (2006), 867-887.

40. Q. LIU, 'Schur algebras of classical groups II', preprint 2007, University of Koeln.

41. I.G. Macdonald, Symmetric functions and Hall polynomials, Second Edition, Oxford Mathematical Monographs (The Clarendon Press, Oxford University Press, New York, 1995).

42. A. Mathas, Iwahori-Hecke algebras and Schur algebras of the symmetric group, University lecture series, 15 (American Mathematical Society, Providence, R.I., 1999).

43. E. Murphy, 'On the representation theory of the symmetric groups and associated Hecke algebras', $J$. Algebra 152 (1992) 492-513.

44. E. MurPhy, 'The representations of Hecke algebras of type $A_{n}$ ', J. Algebra 173 (1995) 97-121.

45. S. Oenms, 'Centralizer coalgebras, FRT-construction, and symplectic monoids', J. Algebra (1) 244 (2001) $19-44$.

46. I. Schur, 'Über die rationalen Darstellungen der allgemeinen linearen Gruppe', (1927). Reprinted in I. Schur, Gesammelte Abhandlungen, Vol. III, pp. 68-85 (Springer-Verlag, Berlin, 1973).

47. H. WeYL, The classical groups, their invariants and representations (Princeton University Press, 1946).

48. H. WenzL, 'On the structure of Brauer's centralizer algebras', Ann. of Math. 128 (1988) 173-193.

\author{
Stephen Doty \\ Department of Mathematics and Statistics \\ Loyola University Chicago \\ 6525 North Sheridan Road \\ Chicago IL 60626 \\ USA \\ doty@math.luc.edu
}

Jun $\mathrm{Hu}$

Department of Applied Mathematics

Beijing Institute of Technology

Beijing 100081

P.R. China

junhu303@yahoo.com.cn 